\title{
Antifungal benzimidazoles disrupt vasculature by targeting one of nine $\beta$-tubulins
}

\author{
Riddhiman K. Garge ${ }^{1, \#}$, Hye Ji Cha ${ }^{1,2, \#}$, Chanjae Lee ${ }^{1}$, Jimmy D. Gollihar ${ }^{1,3}$, Aashiq H.
} Kachroo $^{4}$, John B. Wallingford ${ }^{1}$, Edward M. Marcotte ${ }^{1}$

${ }^{1}$ Department of Molecular Biosciences, The University of Texas at Austin, Austin, TX 78712, USA

${ }^{2}$ Division of Hematology/Oncology, Boston Children's Hospital and Department of Pediatric Oncology, Dana-Farber Cancer Institute, Harvard Stem Cell Institute, Harvard Medical School, Boston, MA 02115, USA

${ }^{3}$ US Army Research Laboratory - South, Austin, TX, USA

${ }^{4}$ The Department of Biology, Centre for Applied Synthetic Biology, Concordia University, Montreal QC H4B 1R6, Canada

${ }^{\#}$ These authors contributed equally to this work Correspondence: marcotte@icmb.utexas.edu, wallingford@austin.utexas.edu 


\section{ABSTRACT}

2 Thiabendazole (TBZ) is an FDA-approved benzimidazole widely used for its antifungal and 3 antihelminthic properties. We showed previously that TBZ is also a potent vascular disrupting

4 agent and inhibits angiogenesis at the tissue level by dissociating vascular endothelial cells in 5 newly formed blood vessels. Here, we uncover TBZ's molecular target and mechanism of action. 6 Using human cell culture, molecular modeling, and humanized yeast, we find that TBZ selectively 7 targets only 1 of 9 human $\beta$-tubulin isotypes (TUBB8) to specifically disrupt endothelial cell 8 microtubules. By leveraging epidemiological pesticide resistance data and mining chemical 9 features of commercially used benzimidazoles, we discover that a broader class of benzimidazole 10 compounds, in extensive use for 50 years, also potently disrupt immature blood vessels and inhibit 11 angiogenesis. Thus, besides identifying the molecular mechanism of benzimidazole-mediated 12 vascular disruption, this study presents evidence relevant to the widespread use of these 13 compounds while offering potential new clinical applications. 


\section{INTRODUCTION}

15 The vascular system is built by the combination of de novo formation of blood vessels by vasculogenesis and the sprouting of new vessels from existing vessels via angiogenesis ${ }^{1,2}$.

17 Imbalances in angiogenesis underlie a variety of physiological and pathological defects, including

18 ischemic, inflammatory, and immune disorders ${ }^{1,3,4}$. Indeed, angiogenesis is central to tumor malignancy and cancer progression, as new blood vessels must be established to supply oxygen and nutrients to the growing tumor. Accordingly, inhibition of angiogenesis is now a wellrecognized therapeutic avenue ${ }^{1-5}$. Defined angiogenesis inhibitors such as Avastin (FDA approved since 2004) are now in wide use in the clinic and, over the past 30 years, several dozen drugs have been approved or entered clinical trials as angiogenesis inhibitors ${ }^{5-9}$.

In recent years, a new class of anti-vascular drugs, termed vascular disrupting agents (VDAs), have gained attention as potential alternative therapeutics operating by distinct mechanisms ${ }^{10-13}$. Unlike angiogenesis inhibitors which selectively prevent the formation of new blood vessels, VDAs function by dismantling existing vasculature, making them potentially effective for therapies beyond cancer, for example in the treatment or control of macular degeneration and diabetic retinopathies $^{14,15}$. While several VDAs have shown therapeutic potential, none have yet been approved, with several candidates still in clinical trials ${ }^{11,16,17}$.

Given the lengthy approval process, the failure of many drugs to succeed in clinical trials, and the high costs involved with developing new compounds, drug repurposing offers an attractive alternative for developing new therapies more quickly. We recently developed strategies to exploit data from diverse model organisms to identify both deeply conserved genetic networks as well as small molecules that may manipulate them ${ }^{18-20}$. This effort identified thiabendazole (TBZ) as both a novel angiogenesis inhibitor and $\mathrm{VDA}^{20}$.

TBZ is one of a large class of biologically active benzimidazole compounds that are widely used commercially or clinically, with applications ranging from photographic emulsions and circuit board manufacturing, to serving as one of the most common heterocyclic ring systems used for small molecule drugs ${ }^{21}$. The FDA approved TBZ in 1967 for human use for treating systemic

41 fungal and helminthic infections, but it is more widely used in veterinary settings and in

42 agricultural pesticides and preservatives. However, we found that TBZ also possesses potent 
43 vascular disrupting ability, demonstrated in vitro in human cell culture and in vivo in mice and

44 frogs, including for retarding tumor growth and reducing intratumoral vessel density in preclinical murine xenograft models ${ }^{20}$.

Several other VDAs have been reported to collapse the vasculature by inhibiting microtubule 47 polymerization dynamics via binding $\beta$-tubulin ${ }^{11,16}$. Indeed, though the basis for TBZ's vascular 48 disrupting action is unknown, it is proposed that TBZ's fungicidal action is mediated via disrupting 49 fungal microtubule assembly and dynamics ${ }^{22,23}$. In particular, mutations in $\beta$-tubulin have been 50 frequently found to confer resistance to TBZ in parasitic/invasive fungal and nematode species ${ }^{21-}$

513 . However, in humans, TBZ does not generally disrupt cell growth, and even in human umbilical 52 vein endothelial (HUVEC) cells, while it somewhat reduced tubulin protein abundance it did not 53 elicit gross defects in the microtubule cytoskeleton ${ }^{20}$. At angiogenesis-inhibiting doses, the overall 54 development of TBZ treated animals is normal ${ }^{18}$, consistent with TBZ's safety record in humans 55 and veterinary settings ${ }^{24}$. Therefore, we hypothesized that only certain types of human cells, such 56 as subsets of endothelial cells involved in forming the vasculature, might be uniquely susceptible 57 to TBZ.

58 Here, we experimentally determined TBZ's specific molecular target and cellular mechanism of 59 vascular disrupting activity. We find that TBZ disrupts microtubule growth, with increased 60 potency in endothelial cells. Using predictive molecular modeling, human cell culture, and 61 humanized yeast, we find TBZ predominantly targets only one of nine human $\beta$-tubulins, 62 suggesting an explanation for its cell-type specificity. Finally, based on epidemiological data 63 mining and chemical structures, we discovered that a larger family of benzimidazoles - in clinical 64 and commercial use for $>50$ years - all act as VDAs, disrupting the vasculature in a vertebrate 65 animal model. These newly discovered VDAs include two World Health Organization (WHO) 66 antihelminthics (albendazole and mebendazole) administered for the treatment of human intestinal 67 infections, one broad-spectrum antifungal/antihelminthic (fenbendazole) used to treat farm animal 68 infections, and two banned pesticides (benomyl and carbendazim) used to prevent wild fungal and 69 nematode mediated crop destruction. Knowledge of their vascular disrupting activities should thus 70 inform their use in at-risk individuals (such as during pregnancy) and opens new clinical 71 applications for these compounds. 


\section{RESULTS}

\section{Thiabendazole disrupts microtubule plus ends in endothelial cells}

75 Thiabendazole exhibits broad-spectrum activity against fungal and nematode crop pests ${ }^{25}$, but 76 prior to demonstration of its VDA activity, it was generally thought to lack activity in tetrapods ${ }^{24}$.

77 Its binding target and mechanism of vascular action remains poorly understood ${ }^{26}$, although in vitro

78 studies have suggested various benzimidazole compounds inhibit cell growth by interfering with 79 microtubule polymerization ${ }^{23,26-28}$. Benzimidazole suppressor screens in both Saccharomyces 80 cerevisiae and Caenorhabditis elegans have independently identified resistance mutations 81 occuring in $\beta$-tubulin genes, giving some insight into the binding site ${ }^{29,30}$. The case for a $\beta$-tubulin 82 binding site is strengthened by numerous animal and agricultural studies also demonstrating 83 resistance mutations arising repeatedly and independently across multiple parasitic nematode and 84 fungal species infecting farm livestock and crops $^{22,27,31-43}$.

85 To examine the effects of the microtubule cytoskeleton and dynamics in the presence of TBZ, we 86 examined the localization of GFP-tagged EB1, which labels growing microtubule plus ends and 87 provides a proxy for microtubule dynamics in both endothelial (HUVEC) and non-endothelial 88 (NIH3T3) human cells. Despite the grossly normal architecture of microtubules in TBZ-treated 89 HUVECs, TBZ significantly reduced the accumulation of EB1 at microtubule plus ends (Fig. 1A', 90 B') as compared to its control (Fig. 1A, B). Importantly, and consistent with the overall normal 91 morphology and patterning of TBZ-treated embryos ${ }^{20}$, we found that TBZ had a substantially less 92 robust effect on EB1 accumulation at microtubule plus ends in fibroblasts as compared to 93 endothelial cells (Fig. 1C-E). These data are consistent with, and provide new insights into, 94 previous studies showing that TBZ's interaction with tubulin interferes with microtubule 95 polymerization in nematodes and fungi ${ }^{44,45}$.

\section{Thiabendazole selectively targets TUBB8 among human $\beta$-tubulins}

97 Three commonly observed mutations in fungal and nematode $\beta$-tubulins (F200Y, E198A, and 98 F167Y) confer resistance to TBZ (Fig. 2A, B), suggesting that its binding site is in the vicinity of 99 these residues ${ }^{22,27,31-43}$ (File S1). Based on the previously observed benzimidazole suppressor 
mutations, we used 3D structural modeling to evaluate TBZ's potential binding sites in a fungal $\beta$-tubulin. We first constructed 3D homology models of the Schizosaccharomyces pombe (fission yeast) wild-type and TBZ-resistant F200Y $\beta$-tubulins, based on the previously determined Ovis aries $\beta$-tubulin crystal structures (PDB: $3 \mathrm{UT}^{46}$ and $3 \mathrm{~N}^{2} \mathrm{G}^{47}$ ) as templates. We computationally refined the structures and then evaluated potential binding modes of TBZ, as detailed in the Methods, using computational docking algorithms to localize TBZ's potential binding sites within the fungal $\beta$-tubulin structures (Fig. S1). We identified a binding site around F200 to be the most probable (File S2). We found that the preferred binding conformations of TBZ in both models (Fig. S1) situated close to (but distinct from) the colchicine binding site. These observations were in strong agreement with computational predictions made on parasitic $\beta$-tubulins binding benzimidazoles $^{27}$ and recent crystal structures of other benzimidazole derivatives binding to bovine brain $\beta$-tubulins ${ }^{48}$.

On measuring the polar contacts and clashing energies of TBZ with tubulin, we found that the wild-type $\beta$-tubulin bound to TBZ more favorably with contact energy $(-9.9 \mathrm{kcal} / \mathrm{mol})$ as compared to its F200Y counterpart, which showed unfavorable repulsions $(+27.6 \mathrm{kcal} / \mathrm{mol})(\mathbf{F i l e} \mathbf{S 2}$, S3). For the wild-type protein, TBZ's polar contacts included E198 and Q134 (Fig. 2C, S1). Arenehydrogen interactions between the drug and protein included contributions from F200, L250, and L253. However, for our F200Y mutant, repulsion was observed in our fixed ligand experiments predominantly caused by unfavorable contacts made with Y200, F240, L250, and L253 (Fig. S1). Our analyses suggest F200Y likely forms a hydrogen bond to E198 in the TBZ-resistant mutant, thus constricting the pocket and occluding binding.

121 Unlike fungi, tetrapods have multiple $\beta$-tubulin isotypes (here, we use the term isotype to denote 122 the protein products of paralogous genes, in accordance with prior tubulin literature), and their 123 expression varies in different cells and tissue types. For example, human tubulin $\beta I$ 124 (TUBB/TUBB5) is constitutively expressed in many cells and tissues, whereas $\beta$ III (TUBB3) is 125 exclusively enriched in neurons and the brain $^{49,50}$. The specific roles of different $\beta$-tubulin isotypes 126 are not yet fully understood, but recent studies indicate that their sequence diversity modulates 127 binding affinity to tubulin-binding drugs and influences microtubule dynamics through distinct 128 interactions with molecular motors ${ }^{50,51}$. The recurrence of TBZ resistance mutations at the same 129 three loci across diverse fungi and nematodes (File S1) led us to hypothesize that human $\beta$-tubulin 
isotypes might have differential sensitivities to TBZ by virtue of incorporating resistant residues at positions 167,198 , and 200, potentially explaining both its tissue-specific effects and generally low toxicity in humans. remained conserved across all the human isotypes, positions 198 and 200 were variable (Fig. 2B). Moreover, all human $\beta$-tubulin isotypes except TUBB1 and TUBB8 contain the F200Y resistance mutation. Because TUBB1 also harbors the other commonly observed E198A suppressor (Fig. 2B), TUBB8 is the only human $\beta$-tubulin isotype predicted by sequence to be TBZ-sensitive. Given this variability across isotypes, we next asked how the E198A and F200Y mutations would be expected to affect TBZ's ability to bind at its predicted site in human isotypes.

We first evaluated this hypothesis computationally, by constructing 3D homology models for each of the human $\beta$-tubulin isotypes in the same manner as for the fungal model (see Methods). We then performed induced-fit docking with TBZ across our human $\beta$-tubulin models. Using a TBZwild-type fungal $\beta$-tubulin complex as a template, we docked TBZ into the same pocket in each of the human isotypes and measured protein-ligand interactions in the superimposed structures. In agreement with our primary sequence based predictions, TBZ fit well into the predicted binding pocket of only TUBB1 and TUBB8, which lack the F200Y mutation. Both showed favorable

147 binding energies of -1.8 and $-8.3 \mathrm{kcal} / \mathrm{mol}$, respectively (File S2). The large difference in contact energy among these isotypes could be explained by position 198. In TUBB1, alanine occupies position 198, whereas TUBB8 has glutamate, which contributed heavily to the binding energy in

150 all of our simulations when both F200 and E198 were present. Our data suggest that TBZ binding 151 is stabilized by hydrogen bonds with residues Q134 and E198 in the presence of F200 (Fig. S1, 152 S2). Taken together, our in silico studies predicted that TBZ should strongly bind TUBB8 and 153 weakly bind TUBB1, but should not bind any other human $\beta$-tubulin isotypes.

154 Functional assays in human endothelial cells and humanized yeast confirm TBZ specificity to human TUBB8

Given TBZ's effects on human vascular endothelial cells and in vivo vascular disruption in 157 Xenopus embryos ${ }^{20}$, we wished to test directly if TUBB8-specific binding could explain the compound's effects. We thus asked whether resistance to TBZ could be acquired by simply 
supplying human $\beta$-tubulin isotypes predicted to be resistant. We tested this by two independent assays: (i) by overexpressing specific sensitive or resistant human $\beta$-tubulin isotypes in human endothelial cells and (ii) by humanizing Baker's yeast's $\beta$-tubulin TUB2 to enable assays of individual human $\beta$-tubulin isotypes.

To test if microtubule dynamics in human cells could be significantly restored by supplying resistant $\beta$-tubulin isotypes, we singly transfected HUVEC cells with plasmids overexpressing either TUBB4 or TUBB8 and assayed microtubule dynamics by measuring the comet lengths of end-binding protein EB3 (Fig. 3A). Compared to untransfected HUVECs, we saw that overexpressing TUBB4 significantly rescued the decrease in comet length observed in TBZtreated cells (Fig. 3B). Transfection with $T U B B 8$, by contrast, had no effect (Fig. 3B). The differences became very significant after 30 minutes of exposure (Fig. 3B).

As an independent assay of TBZ action on human tubulins, we turned to humanized yeast, as our previous work showed that of the nine human $\beta$-tubulins, only TUBB4 and TUBB8 could 172 functionally replace TUB2 in Saccharomyces cerevisiae ${ }^{52}$. From our modeling and docking data, 173 we hypothesized that yeast strains humanized with TUBB8 would be susceptible to TBZ while 174 humanizing with TUBB4 would confer TBZ resistance. Saccharomyces cerevisiae possesses $2 \alpha-$ 175 tubulins (TUB1 and TUB3) that interact with $T U B 2$ to form tubulin heterodimers, which in turn 176 oligomerize to form microtubules. Wild-type BY4741 haploid strains are TBZ-resistant. However, 177 previous studies have shown that on deleting $T U B 3$, yeast strains become susceptible to 178 benzimidazoles ${ }^{53}$ likely due to reduced overall $\alpha$-tubulin stoichiometry or possibly by TBZ 179 occluding TUB2's dimerization with $T U B 1$ but not $T U B 3$. Therefore, we performed all our yeast replacement assays in a $t u b 3 \Delta$ background, which yielded a clear growth defect in the presence of TBZ (Fig. S3). In order to test the effect of TBZ on human $\beta$-tubulin isotypes TUBB4 and TUBB8, we used CRISPR/Cas9 to construct yeast strains with these human isotypes in place of the endogenous TUB2 and tested them in the presence or the absence of the drug (Fig. 3A). We found that strains possessing wild-type $T U B 2$ and human TUBB8 exhibited slow growth in the presence of TBZ (at conc. $20 \mu \mathrm{g} / \mathrm{ml}$ ). By contrast, the strain humanized with TUBB4, which is predicted to be resistant to TBZ, grew normally in the presence of TBZ (Fig. 3C, S3A). 
187 Together with our in silico docking data, our results in HUVECs and humanized yeast indicate 188 that TUBB8 is uniquely TBZ-sensitive, suggesting in turn that vascular endothelial cells are 189 selectively sensitive to its loss.

\section{Benzimidazole resistance patterns and chemical similarities suggest additional VDAs}

191 Given the plethora of fungal and nematode studies on benzimidazole pesticide resistance in 192 agriculture $22,33-36,38,40-44,54-71$ (Fig. 4A), we reasoned that TBZ's molecular mechanism may extend 193 to other commercially used benzimidazole compounds. Indeed, based on our experiments, a simple 194 epidemiological signature should be sufficient to identify other pesticides that likely to function as 195 vascular disrupting agents and angiogenesis inhibitors: (i) the compounds should be selectively 196 toxic to fungal and nematode clades but demonstrate low toxicity in tetrapods, and (ii) sensitive 197 species should specifically gain benzimidazole resistance from F167Y, E198A or F200Y $\beta$-tubulin 198 mutations.

In order to understand how extensively distributed benzimidazole resistance was, we mined $\sim 40$ years of literature to identify reported cases of pesticide resistant species seen in wild and parasitic nematodes and fungi. Benzimidazole resistance is a global phenomenon (Fig. 4A); across 9 major commercial benzimidazole-based pesticides, we found multiple independent instances of reported resistance across 27 (12 nematodes and 15 fungal) parasitic species (File S1), all of which exhibited at least 1 of the 3 signature $\beta$-tubulin mutations. These widespread patterns of benzimidazole pesticide resistance suggested at least 9 new candidate VDAs.

As a complement to the epidemiological data, we also considered chemical properties by asking if pesticide benzimidazoles shared similar chemical feature profiles relative to other benzimidazoles.

208 We curated $>80$ commercially available compounds in the benzimidazole class spanning a diverse 209 range including pesticides, fungicides, therapeutics, and preservatives. Upon hierarchical 210 clustering of these benzimidazoles based on their chemical properties computed from JOELib's 211 features matrix ${ }^{72,73}$ (File S5), we found that pesticide benzimidazoles generally shared similar 212 chemical properties and clustered together (Fig. 4B). 
214 We next tested if pesticides exhibiting the epidemiological signature and clustering in the same

215 clades by virtue of their chemical features would also specifically inhibit TUBB8 and function as

216 VDAs. We selected 12 commercially used benzimidazole compounds across 2 clusters (Fig. 4B).

217 Our list included 2 anthelmintics, both World Health Organization essential medicines

218 (albendazole and mebendazole) prescribed to treat broad-spectrum human intestinal nematode 219 infections; fenbendazole, an anthelmintic prescribed specifically for animals against 220 gastrointestinal nematode parasites; 2 currently banned pesticides, benomyl and carbendazim, 221 formerly used in agriculture; triclabendazole, specifically used to treat liver fluke infections; and 2225 proton-pump inhibitors (esomeprazole, lansoprazole, omeprazole, pantoprazole, and 223 rabeprazole) used to treat gastrointestinal and stomach acid disorders. The latter set were from a 224 different clade and did not exhibit the epidemiological signature, serving as negative controls.

225 We first took advantage of our humanized yeast strains to rapidly discriminate TUBB8-specific 226 inhibition from general $\beta$-tubulin inhibition. We found that 5 of the 12 compounds tested 227 selectively inhibited TUBB8, as evidenced by the growth profiles observed for the humanized 228 strains when cultured in the presence of the drugs (Fig. 5, S4). Notably, none of the 5 proton pump 229 inhibitors or colchicine exhibited any tubulin inhibition (Fig. S4, S5A), confirming the specificity 230 of the epidemiological signature as a predictor of TUBB8 inhibition. In contrast, triclabendazole 231 was generally toxic, behaving as a pan-isotype inhibitor (Fig. S5B).

232 Testing the 5 positive TUBB8-inhibiting compounds in Xenopus laevis embryos showed strong 233 vascular disrupting activity for all 5 compounds (Fig. 5). As we observed previously for TBZ ${ }^{20}$, 234 the gross morphology of the treated embryos was largely normal (Fig. 5). Thus, this broader class 235 of benzimidazoles do in fact generally act as vascular disrupting agents in vertebrates.

237 DISCUSSION

238 In the $>30$ years of therapeutic research efforts in the angiogenesis field only a highly restricted 239 set of drugs have yet been approved ${ }^{11}$. Given the frequent failure to successfully make it through 240 clinical trials and the high costs and lengthy process associated with developing new compounds, 241 drug repurposing can offer efficient alternatives in developing new patient therapies with 
242 accelerated timeframes. This study represents a rather unconventional path to drug repurposing,

243 leveraging a combination of model organisms, humanized yeast, cell culture, molecular modeling,

244 and epidemiological data mining to determine TBZ's molecular target and mechanism of vascular

245 action. Indeed, TBZ was initially identified as a VDA and angiogenesis inhibitor by using a

246 Baker's yeast model of angiogenesis discovered in a computational search for orthologous

247 phenotypes, or phenologs, aimed at exploiting deep evolutionary conservation to prioritize yeast

248 processes relevant to human diseases ${ }^{18-20}$. Although obviously lacking blood vessels and a

249 circulatory system, yeast nonetheless retains conserved biological pathways and processes relevant

250 to vertebrate angiogenesis genes, and it was on the basis of these conserved processes that the

251 antifungal compound TBZ was initially suspected, later confirmed, to be an angiogenesis

252 inhibitor $^{20}$.

253 While TBZ somewhat reduced the abundance of tubulin proteins in human cells ${ }^{20}$, at angiogenesis-

254 inhibiting doses, the overall morphology of TBZ treated animals was normal, suggesting that only

255 certain cell types, specifically those endothelial cells involved in forming the vasculature, might

256 be uniquely susceptible to TBZ. Here, we find that TBZ does indeed specifically modulate the

257 microtubules in vascular endothelial cells. Several currently identified microtubule targeting drugs

258 have been reported to interfere with polymerization dynamics by binding $\beta$-tubulin ${ }^{11,16}$ close to or

259 at the colchicine binding site. Building on previous work ${ }^{27,48}$, our in silico modeling results suggest

260 that TBZ's binding site, while in close proximity to the colchicine binding site, is distinct from it,

261 thereby uncovering a novel $\beta$-tubulin effector site likely specific to other benzimidazoles and TBZ

262 analogs.

263 In contrast to $\beta$-tubulin anticancer drugs, which have largely shown pan-isotype activity, to our

264 knowledge, this study presents an unusual case of isotype-specific drug targeting in the $\beta$-tubulin

265 gene family. Fungal suppressor studies on benzimidazole resistance have repeatedly found 266 resistant mutations in $\beta$-tubulin; we found that 8 of 9 human $\beta$-tubulins natively harbor the same

267 suppressor mutations and consequently exhibit unfavourable steric clashes interfering with TBZ

268 binding. We demonstrate both via human cell culture microtubule assays and humanized yeast

269 drug sensitivity tests that TBZ selectively targets only TUBB8 among the nine human $\beta$-tubulins,

270 thus disrupting microtubule dynamics and reducing end-binding protein accumulation at the plus

271 ends of microtubules in susceptible cells. 
272 With TUBB8 thus acting as the specific target, it follows that of all human cell types, vascular 273 endothelial cells must in turn be particularly sensitive to inhibition of TUBB8, leading to selective 274 disruption of the vasculature relative to other human tissues. It remains to be seen why TBZ's 275 vascular disrupting activity is restricted to immature or newly forming blood vessels, but we 276 speculate that this subset of the vasculature lacks reinforcing cell-cell contacts typical of larger, 277 more established vasculature, leading to greater sensitivity to TBZ-induced microtubule 278 disruption. As $\beta$-tubulin isotypes tend to be broadly expressed and often substitute for one another 279 in microtubule structures ${ }^{74,75}$, one possibility is that TUBB8 inhibition simply leads to the loss of 280 interactions with endothelial cell-specific components, thus specifically impacting 281 vasculogenesis/angiogenesis. However, gene-gene and gene-drug interactions can often proceed 282 by less obviously direct mechanisms to selectively impact cell types or phenotype penetrance via 283 conditional cell-specific or dosage-dependent synthetic interactions ${ }^{76,77}$. It would thus not be 284 surprising for the consequences of inhibiting TUBB8 in vascular endothelial cells to be similarly 285 indirectly mediated by endothelial cell-specific synthetic interactions. Further experiments 286 characterizing TBZ's selective activity against newly forming/formed vasculature and the 287 vascular-specific roles of TUBB8 in tetrapods could offer valuable insights into the cytoskeletal 288 dynamics underlying vasculogenesis and angiogenesis.

289 Based on chemical properties and signature resistance mutations observed against benzimidazole compounds, we identified a larger class of extensively used fungicides and pesticides that all exhibit vascular disruption activity. While our results suggest possible new clinical applications 292 for these compounds, they also highlight the potential caveats of their use in at-risk populations, 293 especially for the two compounds (albendazole and mebendazole) that are FDA approved for 294 human use. The WHO recommends the use of both albendazole and mebendazole as essential 295 antihelmenthics worldwide for children up to the age of 14 against soil-transmitted helminth 296 infections. Moreover, these compounds are widely used as public health interventions in pregnant 297 women after the first trimester in regions where hookworm and whipworm infections exceed $29820 \%{ }^{78,79}$. While in the US, the risk of mebendazole use during pregnancy has not been assigned, 299 our data add weight to WHO recommendations that these drugs should not be administered in the 300 first trimester of pregnancy and suggest their use be carefully evaluated in patients in which 301 angiogenesis inhibition might pose risks, including using caution later in pregnancy in light of the 302 evidence that the compounds disrupt immature vasculature and might prove harmful to a 
303 developing fetus. Conversely, while efforts in the angiogenesis field have been often motivated

304 towards developing anticancer therapies, the wide use of the compounds discussed here and their

305 FDA-approved status could open alternative paths to treating other angiogenesis and/or vascular

306 related diseases, such as diabetic retinopathy, macular degeneration, and hemangioma. It remains

307 to be seen if other benzimidazoles sharing similar chemical profiles to those tested in our work

308 (such as ciclobendazole, nocodazole, oxibendazole, and oxfendazole) also exhibit vascular 309 disrupting activity.

310 More broadly, our framework of leveraging phenotypic relationships between species and 311 repurposing model organisms to systematically explore drug mechanisms opens new routes for 312 drug repurposing and discovery, and highlights the power of systems biology and evolution-guided 313 approaches in advancing our knowledge of conserved genetic modules and how their disruption 314 manifests in disease. This work also illustrates how duplicated genes diversify their functions and 315 reinforces the therapeutic benefits of finding drugs specific to individual gene family members. 316 As evidenced by the high degree of replaceability of conserved genes from cross-species 317 complementation assays ${ }^{80-82}$, we anticipate that the combination of humanized yeast and 318 phenolog-based disease modeling can be extended beyond vascular disruption to other conserved 319 processes and therapies targeting them.

\section{MATERIALS AND METHODS}

\section{Multiple sequence alignment}

323 Human gene sequences were downloaded from the Uniprot database. The multiple sequence 324 alignment for $S$. pombe, S. cerevisiae, and 9 human $\beta$-tubulin genes was constructed using MAFFT $\mathrm{v} 7^{83}$ and visualized in Geneious v10 (https://www.geneious.com).

327 Homology models of human and fungal $\beta$-tubulins were constructed using as a reference structure 328 the previously determined Ovis aries $\beta$-tubulin crystal structures (PDB: $3 \mathrm{UT} 5$ and $3 \mathrm{~N} 2 \mathrm{G})^{46,47}$. The template was prepared using the Molecular Operating Environment (MOE.09.2014) software 
330 package from Chemical Computing Group. The structure was inspected for anomalies and 331 protonated/charged with the Protonate3D subroutine $(310 \mathrm{~K}, \mathrm{pH} 7.4,0.1 \mathrm{M} \text { salt })^{84}$. The protonated structure was then lightly tethered to reduce significant deviation from the empirically determined coordinates and minimized using the Amber10:EHT forcefield with R-field treatment of electrostatics to an RMS gradient of $0.1 \mathrm{kcal} \mathrm{mol}^{-1} \AA^{-1}$. Homology models of the wild-type fungal $\beta$-tubulin were prepared by creating 25 main chain models with 25 sidechain samples at 298K (625 total) within MOE. Intermediates were refined to an RMS gradient of $1 \mathrm{kcal} \mathrm{mol}^{-1} \AA^{-1}$, scored with the GB/VI methodology, minimized again to an RMS gradient of $0.5 \mathrm{kcal} \mathrm{mol}^{-1} \AA^{-1}$ and protonated. sphere and minimizing the solvent enclosed structure to an RMS gradient of $0.001 \mathrm{kcal} \mathrm{mol}^{-1} \AA^{-1}$. Models were evaluated by calculating Phi-Psi angles and superimposed against the reference structure. Homology models for each human $\beta$-tubulin were prepared similarly, based on generating a total of 625 models and averaging to make a final model for each $\beta$-tubulin isotype.

\section{In silico docking of TBZ into $\beta$-tubulins}

344 Potential binding sites were evaluated using the Site Finder application and recent computational work on benzimidazole binding to parasitic $\beta$-tubulins ${ }^{27,85}$. Conformational variants of TBZ were created in 3-D within MOE. A database of conformations was then used to dock TBZ to the wildtype homology model using induced fit and template similarity protocols. The placement was scored with Triangle Matcher and rescored with London dG. Poses were refined with the Amber10:EHT forcefield with GVBI/WSA dG scoring. Candidate poses were then identified by inspecting polar contacts. Geometry optimization was carried out with MOPAC 7.0 using AM1. Conformational analysis of the bound structure was evaluated with LowModeMD ${ }^{86}$. 2-D contact maps were created using Ligand Interactions ${ }^{87}$.

\section{Cell culture}

354 HUVEC cells were purchased from Clonetics and were used between passages 4 and 9. HUVECs were cultured on $0.1 \%$ gelatin-coated (Sigma) plates in endothelial growth medium-2 (EGM-2; Clonetics) in tissue culture flasks at $37{ }^{\circ} \mathrm{C}$ in a humidified atmosphere of $5 \% \mathrm{CO}_{2}$. NIH-3T3 cells

357 were obtained from Vishy Iyer at the University of Texas at Austin and cultured in Dulbecco's 


\section{Immunohistochemistry}

360 Cell lines were cultured in 6-well plates and treated with thiabendazole dissolved in 1\% DMSO.

361 Control cells received 1\% DMSO. After $24 \mathrm{~h}$, cells were fixed with methanol at $-20{ }^{\circ} \mathrm{C}$ for 10 min 362 and subsequently with 4\% paraformaldehyde in PBS at room temperature for $10 \mathrm{~min}$. Cell 363 membranes were permeabilized with $0.2 \%$ Triton X-100 in PBS, and nonspecific antibody binding 364 sites were blocked with $5 \%$ goat serum for $1 \mathrm{~h}$ at room temperature. Cells were incubated with 365 primary antibodies to EB1 (BD Bioscience) and $\alpha$-tubulin (Sigma) at $4{ }^{\circ} \mathrm{C}$ overnight. After 366 washing with PBST, primary antibodies were detected by Alexa Fluor-488 or 555 goat anti-rabbit 367 or mouse immunoglobulin (IgG). 4',6-Diamidino-2-phenylindole (DAPI dye, Sigma) was added 368 as needed to visualize nuclei.

\section{Cell transfection and perfusion}

370 EB3-eGFP cDNA obtained from Anna Akhmanova was cloned into the vector CS2+ ${ }^{88}$. TUBB4 371 (Origene, RG203945) and TUBB8 (Origene, RG213889) cDNAs were purchased and cloned into 372 the vector CS107-RFP-3Stop. HUVEC cells were transfected by nucleofection (Lonza) according 373 to the manufacturer's instructions. To analyze the effect of TBZ in living cells, we used a closed 374 perfusion system (POC-R2, Pecon) connected to a peristaltic pump (Ismatec). 1\% DMSO, $250 \mu \mathrm{M}$ 375 TBZ or $1 \%$ DMSO diluted in EBM-2 medium was flowed at $100 \mu 1 / \mathrm{min}$ rate for the indicated 376 times.

\section{Western blotting}

378 HUVECs were cultured in 6-well plates and treated with 1\% DMSO or 1\% DMSO, $250 \mu \mathrm{M}$ TBZ 379 for 24 hours. Cells were lysed in cell lysis buffer (Cell Signaling Technology) containing $1 \mathrm{mM}$ 380 PMSF and analyzed by SDS-PAGE and western blotting using anti-EB1 (BD Bioscience) or antiEB3 (Millipore) or anti-Clip170 (Santa Cruz) antibodies.

\section{Imaging and image analysis}

383 Immunohistochemistry experiments, live HUVECs, and live KDR:GFP transgenic Xenopus laevis 384 were imaged using an inverted Zeiss LSM5 Pascal and Zeiss LSM700 confocal microscope, and 385 super-resolution structured illumination (SR-SIM) combined with Zeiss LSM710 microscope. 
Comet lengths were measured using the software Fiji. Confocal images were cropped and enhanced in Adobe Illustrator and Adobe Photoshop for the compilation of figures.

\section{Benzimidazole clustering analysis}

81 commercially used benzimidazole compounds spanning a wide range of classes were curated from PubChem ${ }^{89}$. JOElib (http://joelib.sourceforge.net), OpenBabel ${ }^{90}$, and Chem Mine features were computed using ChemMine tools ${ }^{73}$. Heatmaps were visualized using Morpheus 392 (https://software.broadinstitute.org/morpheus). Clustergrams were generated by hierarchical clustering on the one minus Pearson correlation coefficient with average linkage.

\section{Humanizing yeast $\beta$-tubulin using CRISPR-Cas9}

The human TUBB4 and TUBB8 open reading frames were integrated chromosomally (from start to stop codon) into Saccharomyces cerevisiae in place of the endogenous TUB2 open reading frame using CRISPR/Cas9 genome editing as described in Akhmetov et al. ${ }^{91}$. Two sgRNAs were designed targeting the yeast TUB2 locus using the Geneious (v10.2.6) CRISPR-Cas9 tools suite, purchased as oligos from IDT, and cloned into yeast CRISPR-K/O vectors using the yeast toolkit

$400(\text { YTK })^{92}$ to express a synthetic guide RNA sequence, Cas9 nuclease, and a selectable marker 401 (URA3) ${ }^{52,93}$. Repair templates were constructed by PCR amplification of the human $\beta$-tubulin ORF 402 (from the human ORFeome ${ }^{94}$ ) flanked by 75 bp of target chromosomal boundary at the TUB2 403 locus to facilitate recombination via homology directed repair. BY4741 (S288C) yeast strains were 404 co-transformed with the CRISPR/Cas9 vector and repair template using Zymo Research Frozen405 EZ Yeast Transformation II Kit. Transformants were selected on SC-URA media. Surviving 406 colonies were screened by colony PCR, and Sanger sequenced to confirm replacement.

\section{Humanized yeast growth assays}

408 Assayed benzimidazole compounds were all dissolved in 100\% DMSO to prepare stock solutions 409 of 5 or $10 \mathrm{mg} / \mathrm{ml}$ based on solubility. Candidate VDA compounds were titrated in ranges of 5$4101000 \mu \mathrm{g} / \mathrm{ml}$ into growth medium depending on solubility (Fig. S5 lists specific concentrations) for 411 subsequent growth assays. Liquid growth assays were performed in triplicate in 96-well format 412 using a Biotek Synergy HT incubating spectrophotometer. Humanized tubulin strains were pre- 
413 cultured to saturation in YPD and diluted into $150 \mu \mathrm{L}$ of media to have $0.05-0.1 \times 10^{7}$ cells $/ \mathrm{ml}$.

414 Assays were typically run for $48 \mathrm{hrs}$ with absorbance measured every $15 \mathrm{~min}$.

Xenopus embryo manipulations and VDA assays

416 Xenopus embryos were reared in 1/3× Marc's modified Ringer's (MMR) solution. Each drug was

417 treated to embryos from stage 31 until stage 38 with $10 \mu \mathrm{g} / \mathrm{ml}$ or $20 \mu \mathrm{g} / \mathrm{ml}$ in $1 \%$ DMSO diluted

418 in 1/3X MMR. Embryos were fixed at stage 38 with MEMFA, and whole-mount in situ 419 hybridization for erg was performed as described in Sive et al. ${ }^{87}$.

\section{ACKNOWLEDGMENTS}

422 The authors thank Andrew Ellington for critical feedback and discussions. This research was 423 funded by the American Heart Association Predoctoral fellowship (\#18PRE34060258) to R.K.G., 424 Army Research Office (W911NF-12-1-0390) to J.D.G., Natural Sciences and Engineering 425 Research Council (NSERC) of Canada Discovery grant (RGPIN-2018-05089), CRC Tier 2 426 (NSERC/CRSNG-950-231904), and the Canada Foundation for Innovation and Québec Ministère 427 de l'Économie, de la Science et de l'Innovation (\#37415) to A.H.K., the National Institute of Child 428 Health and Human Development (R01HD099191) to J.B.W., and from the Welch Foundation (F429 1515) and National Institutes of Health (R35 GM122480) to E.M.M..

\section{AUTHOR CONTRIBUTIONS}

432 Conceptualization and methodology, R.K.G., H.J.C., J.D.G., A.H.K., J.B.W., E.M.M.; 433 Computational analyses, R.K.G., H.J.C., J.D.G.; Investigation, R.K.G., H.J.C, C.L., J.D.G.; 434 Formal analysis and visualization, R.K.G., H.J.C., E.M.M.; Writing - R.K.G., H.J.C, J.B.W., 435 E.M.M.

\section{COMPETING INTERESTS}

438 The authors declare no competing interest. 


\section{FIGURE LEGENDS}

440 Figure 1. Thiabendazole (TBZ) significantly reduces EB1 comet length at microtubule plus 441 ends in cultured human cells.

442 Immunohistochemical analysis of $\alpha$-tubulin in two human cell lines using confocal microscopy

443 does not show a definite distinction between 1\% DMSO-treated control $(\mathbf{A}, \mathbf{C})$ and 1\% DMSO, $444250 \mu \mathrm{M}$ TBZ-treated cell lines (B, D), but images from super-resolution microscopy reveal that 445 the accumulation of end-binding (EB) protein 1 at the plus end of microtubules is significantly 446 reduced with TBZ treatment $(\mathbf{B})$ compared to the control (A) in HUVECs. In NIH-3T3 cells, the 447 reduced EB1 comet length following TBZ treatment (D) compared to control (C) is not as 448 pronounced as in HUVECs, as quantified by comet length (E). Scale bars, $20 \mu \mathrm{m}$ in (A) and (C), $4492 \mu \mathrm{m}$ in $\left(\mathrm{A}^{\prime}\right)$ and $\left(\mathrm{C}^{\prime}\right)$.

\section{Figure 2. Uncovering the molecular mechanism of thiabendazole.}

451 (A) TBZ elicits varying activity across different clades of life being toxic to fungal and nematode 452 clades but behaves as a vascular disrupting agent in tetrapods. (B) Of the 9 human $\beta$-tubulins, 8 453 have amino acids at positions 167, 198, and 200 that confer TBZ resistance to fungal tubulins (see 454 Table S1), as seen in a multiple sequence alignment of human and Schizosaccharomyces pombe $455 \beta$-tubulins; only TUBB8 lacks resistance mutations. (C) In silico docking of TBZ (orange) into a 456 homology modeled yeast $\beta$-tubulin 3D structure (see Methods) indicates TBZ is well457 accommodated by a binding pocket in wild-type yeast $N D A 3$ that abuts the 3 major $\beta$-tubulin TBZ 458 resistance mutation sites. In contrast, docking of TBZ into homology models of human TUBB4 459 and TUBB8 indicates the potential for differential binding, with TUBB8 accommodating TBZ 460 whereas, in the case of TUBB4, TBZ is sterically blocked. Polar contacts are illustrated via dashed 461 lines, and residues lining the proposed binding pocket are shown in cyan. Intramolecular hydrogen 462 bonding between E198 and Y200 in TUBB4 reorganizes the geometry of the binding pocket. 463 Residues involved in steric clashing are depicted with a partial mesh surface. (Note that due to 464 steric clashes between TBZ and TUBB4 at the proposed binding pocket, TBZ was superimposed 465 from our binding model to measure interactions). 
Figure 3. TBZ specifically inhibits the human $\beta$-tubulin TUBB8, not TUBB4, in humanized yeast and HUVEC cell culture.

(A) Overview. TBZ's isotype specificity was identified in 2 ways. (Left) Recombinant human $\beta$ tubulins TUBB4 and TUBB8 were individually overexpressed in HUVEC cell culture to monitor comet lengths in the presence of TBZ. (Right) Using humanized yeast wherein yeast TUB2 was singly humanized by either of 2 replaceable human $\beta$-tubulins TUBB4 or TUBB8 to screen for differential sensitivity towards TBZ. (B) Reduced EB3 comet length after 1\% DMSO, $250 \mu \mathrm{M}$ TBZ treatment compared to 1\% DMSO treated control. A. Comet length is similar in EB3, TUBB8 transfected HUVECs compared to EB3 transfected controls expressing native tubulins, but comets are longer in most EB3, TUBB4 transfected cells. B. Comet length is statistically similar between cells treated with 1\% DMSO; however, following 30 minutes of $1 \%$ DMSO, $250 \mu \mathrm{M}$ TBZ treatment TUBB4 transfected cells have significantly longer EB3 comets than HUVECs with TUBB8 or expressing native tubulins. (C) Growth profiles of humanized yeast strains show TBZ's isotype specificity to TUBB8. When grown in the presence of TBZ, Strains carrying the wild-type TUB2 (blue) and human TUBB8 (green) genes are sensitive to TBZ while humanized TUBB4 strains (orange) are resistant. Mean +/- standard deviation indicated by solid lines and shaded boundaries, respectively.

Figure 4. Global trends in benzimidazole resistance mutations and chemical structural similarities suggest numerous potential vascular disrupting agents.

(A) $3 \beta$-tubulin mutations, (F167Y, E198A, F200Y) conferring benzimidazole resistance have been globally observed among parasitic nematode and fungal species. Each icon represents an instance of $\beta$-tubulin suppressor mutations occurring in benzimidazole resistant parasitic fungal or nematode species (See File S1 for list of species showing benzimidazole resistance). (B)

489 Commonly used benzimidazoles hierarchically clustered by their chemical properties suggest new 490 vascular disrupting agents with similar molecular mechanisms to TBZ. (Left) Clustergram of 81 491 widely used benzimidazole compounds spanning a wide range of drug classes grouped by chemical 492 features (See File S5 for the full list of compounds and features analysed). (Right) Zooms of black 493 boxes indicate 3 clades containing TUBB8 specific VDA candidates (top) and proton-pump 494 inhibitors (bottom). 
Figure 5. Commercially used benzimidazole pesticides, antifungals, and antihelminthics are also TUBB8-specific and disrupt vasculature. In situ hybridization of blood vessels (using the erg/flkl probe $^{18}$ ) in Xenopus laevis embryos indicate the disruption of the vasculature caused by the presence of human and animal antihelminthics and broad-spectrum pesticides as compared to the DMSO control. Insets show growth profiles for yeast strains with humanized $\beta$-tubulin TUBB4

500 (orange) and TUBB8 (green) compared to wild-type (blue) when grown in the presence of each 501 compound.

\section{Supplementary Figures}

Figure S1. Homology modeling and in silico docking studies predict the TBZ binding site in binding pocket (A) as opposed to its F200Y mutant (B). 3D structures and 2D contact maps shown on the left and right respectively indicate the steric clashes TBZ faces in the F200Y binding pocket. Cyan meshes (in 3D structures) and red highlights on the ligand (in 2D contact maps) indicate steric clashes in the binding pocket

509 Figure S2. Only TUBB8 favorably binds TBZ among the 9 human $\beta$-tubulins.

510 (A) 2D contact maps highlight ligand interactions between TBZ and TUBB4 (left) or TUBB8 511 (right). TBZ forms polar contacts with residues Q134, E198, F200, and L253 in TUBB8. In

512 TUBB4, reorientation of the proposed binding pocket is observed. Substantial steric clashing is 513 shown in red on TBZ. (B) In silico docking of TBZ into other human $\beta$-tubulin homology models

514 suggests substantial steric clashes due to unfavorable binding pockets among 8/9 human $\beta$-tubulin 515 isotypes. Cyan meshes (in 3D structures) and red highlights on the ligand (in 2D contact maps) 516 indicate steric clashes in the binding pocket

\section{Figure S3. Yeast strains with modified $\beta$-tubulin are differentially sensitive to TBZ.}

518 (A) Deletion of yeast $\alpha$-tubulin TUB3 makes Saccharomyces cerevisiae (Baker's yeast) sensitive 519 to TBZ. (B) Growth profiles of tub3A yeast strains with wild-type TUB2 (blue), humanized $\beta$ 520 tubulins TUBB4 (orange), or TUBB8 (green) in increasing concentrations of TBZ show 521 differential sensitivities to the drug. 


\section{Figure S4. Growth profiles of benzimidazole treated yeast strains.}

523 Plots depict varying doses of albendazole, benomyl, carbendazim, and colchicine. Our data show

524 that colchicine is a pan-isotype inhibitor whereas albendazole, benomyl, and carbendazim show

525 some degree of specificity for TUBB8.

\section{Figure S5. Proton-pump inhibitors do not elicit growth defects in humanized strains.}

527 (A) Yeast strains harboring the beta-tubulin gene TUB2 (blue), TUBB4 (orange), and TUBB8

528 (green) are not inhibited by proton pump inhibitors (drug conc. $40 \mu \mathrm{g} / \mathrm{ml}$ ). (B) Triclabendazole is

529 a pan-isotype $\beta$-tubulin inhibitor inhibiting both wild-type and humanized yeast strains (Left) and

530 lethal to developing Xenopus laevis embryos at stage 38. (Right).

\section{Supplementary information}

533 File S1. Species resistance table curated from literature

$534 \quad$ File S2. Yeast site finder statistics

535 File S3. Docking free energy scores across $\beta$-tubulin isotypes

536 File S4. Yeast wt $\beta$-tubulin induced fit TBZ docking scores

537 File S5. Benzimidazole chemical features 

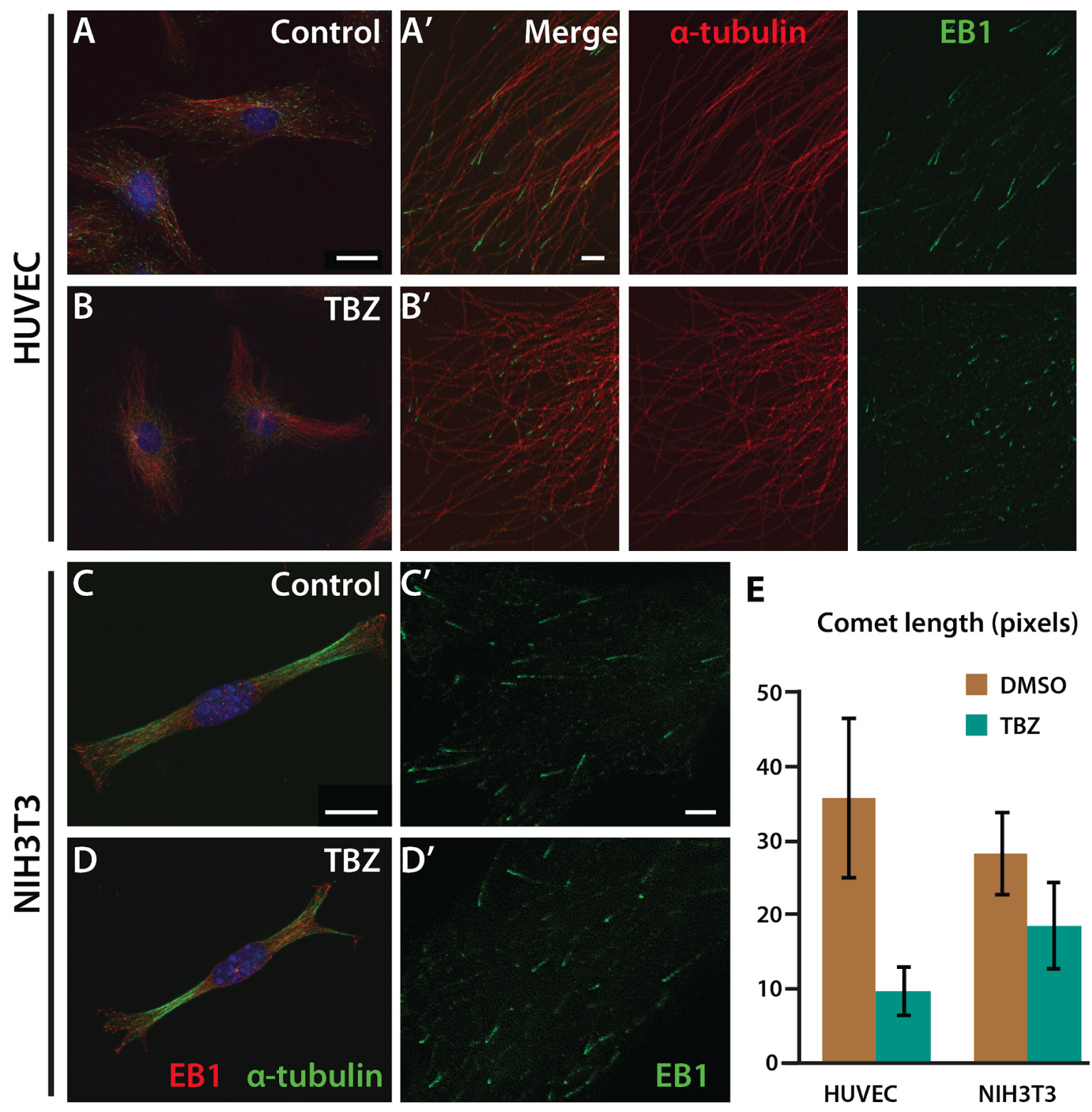

Figure 1. TBZ significantly reduces EB1 comet length at microtubule plus ends in cultured human vascular endothelial cells. 
A

B
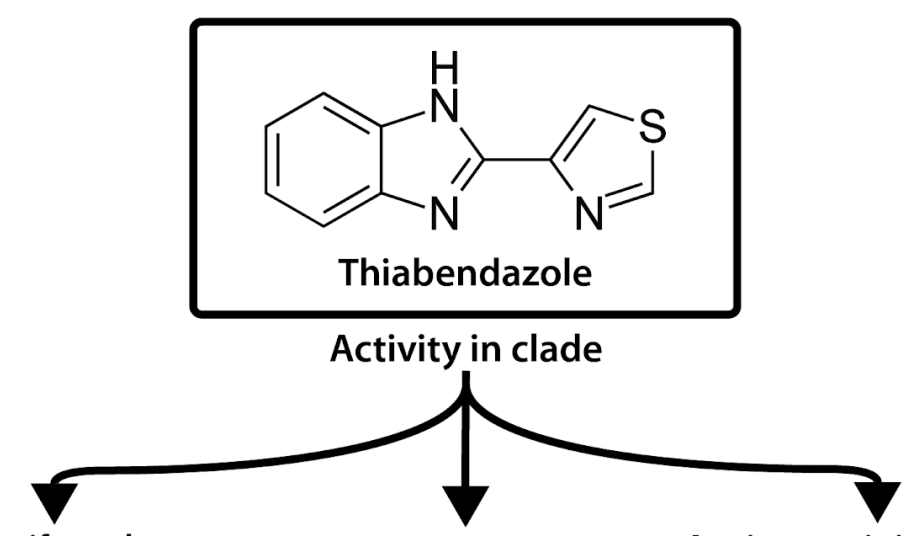

Antifungal

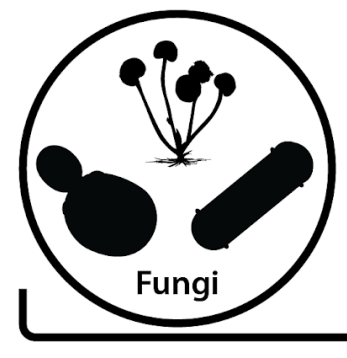

Antihelminthic

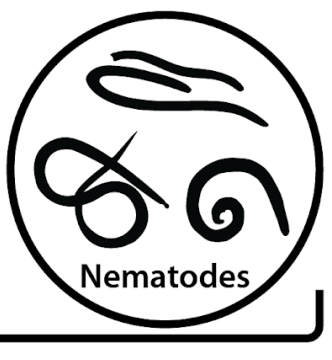

ance mutations
Angiogenesis inhibition \& Vasculature disruption

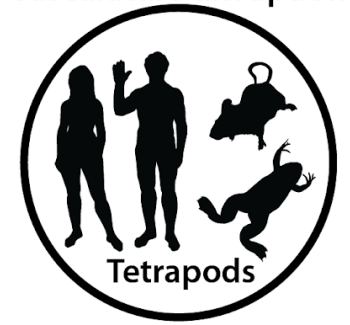

SPNDA3 ATF SVAPAPK S SDTVVEPYNATL SMHQL VENSDETF CI DNEAL SCTUB2 ATF SVLPSPKTSDTVVEPYNATLSVHQLVEHSDETFCIDNEAL

TUBB1 NSF SVMP SPKVSDTVVEPYNAVLS I HQL I ENADACFCI DNEAL TUBB2A NTF SVMP SPKVSDTVVEP YNATLSVHQLVENTDETYSIDNEAL TUBB2B NTF SVMP SP KVSDTVVEPYNATL SVHQL VENTDETYCIDNEAL TUBB2C NTF SVVP SPKVSDTVVEPYNATLSVHQLVENTDETYCIDNEAL

Human TUBB3 NTF SVVPSPKVSDTVVEPYNATLS I HQLVENTDETYCI DNEAL TUBB4 NTF SVVP SPKVSDTVVEPYNATL SVHQLVENTDETYCI DNEAL TUBB5 NTF SVVP SPKVSDTVVEP YNATL SVHQLVENTDETYCIDNEAL

TUBB6 NTF SVMP SPKVSDTVVEPYNATL SVHQLVENTDETYCIDNEAL

TUBB8 NTFSILPSPKVSDTVVEPYNATL SVHQL I ENADET FCI DNEAL

C

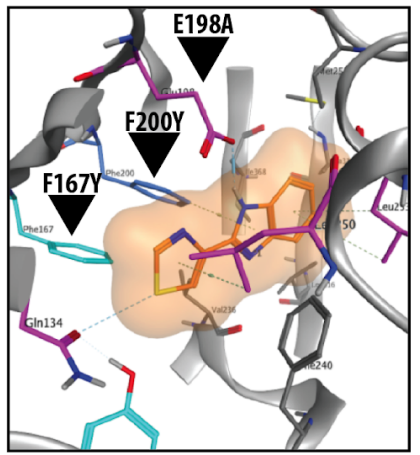

SpNDA3

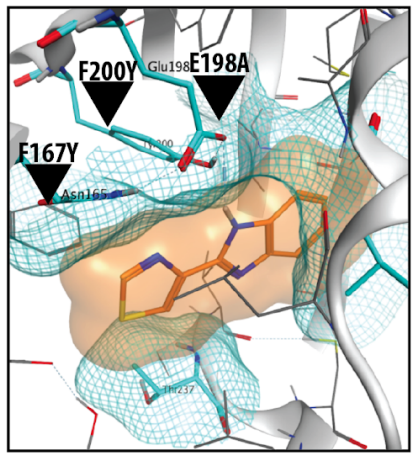

HsTUBB4

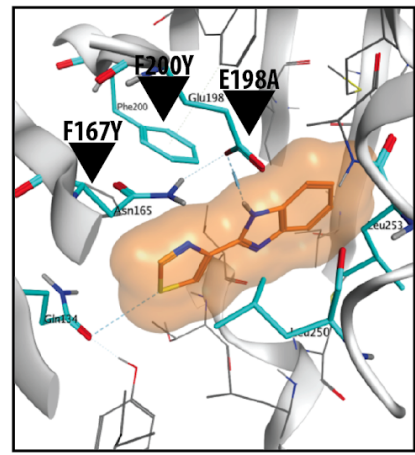

HsTUBB8

Figure 2. Uncovering the molecular mechanism of thiabendazole. 
A

Homo

sapiens

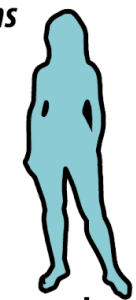

Human

Predicted binding

Replaceable in

B-tubulins

to Thiabendazole

Yeast

\begin{tabular}{|c|c|c|}
\hline HsTUBB1 & * & x \\
\hline HsTUBB2A & 2 & $\mathbf{x}$ \\
\hline HsTUBB2B & s) & $x$ \\
\hline HsTUBB2C & $x$ & $x$ \\
\hline HsTUBB3 & $\boldsymbol{x}$ & 3 \\
\hline HsTUBB4 & $x$ & \\
\hline HsTUBB5 & 3 & 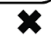 \\
\hline HsTUBB6 & $x$ & $x$ \\
\hline HsTUBB8 & $\checkmark$ & \\
\hline
\end{tabular}
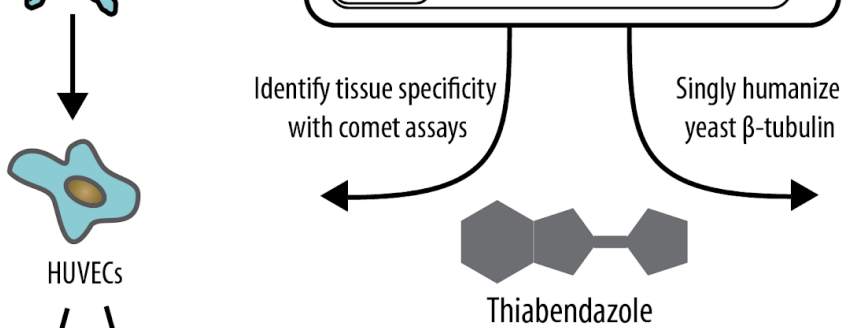

HUVECS
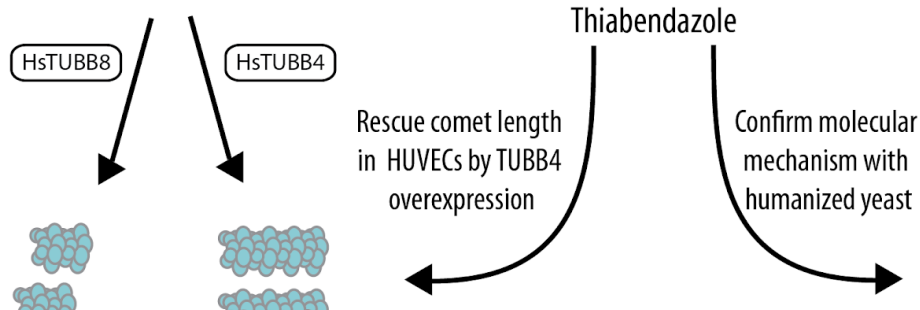

\section{Saccharomyces cerevisiae}
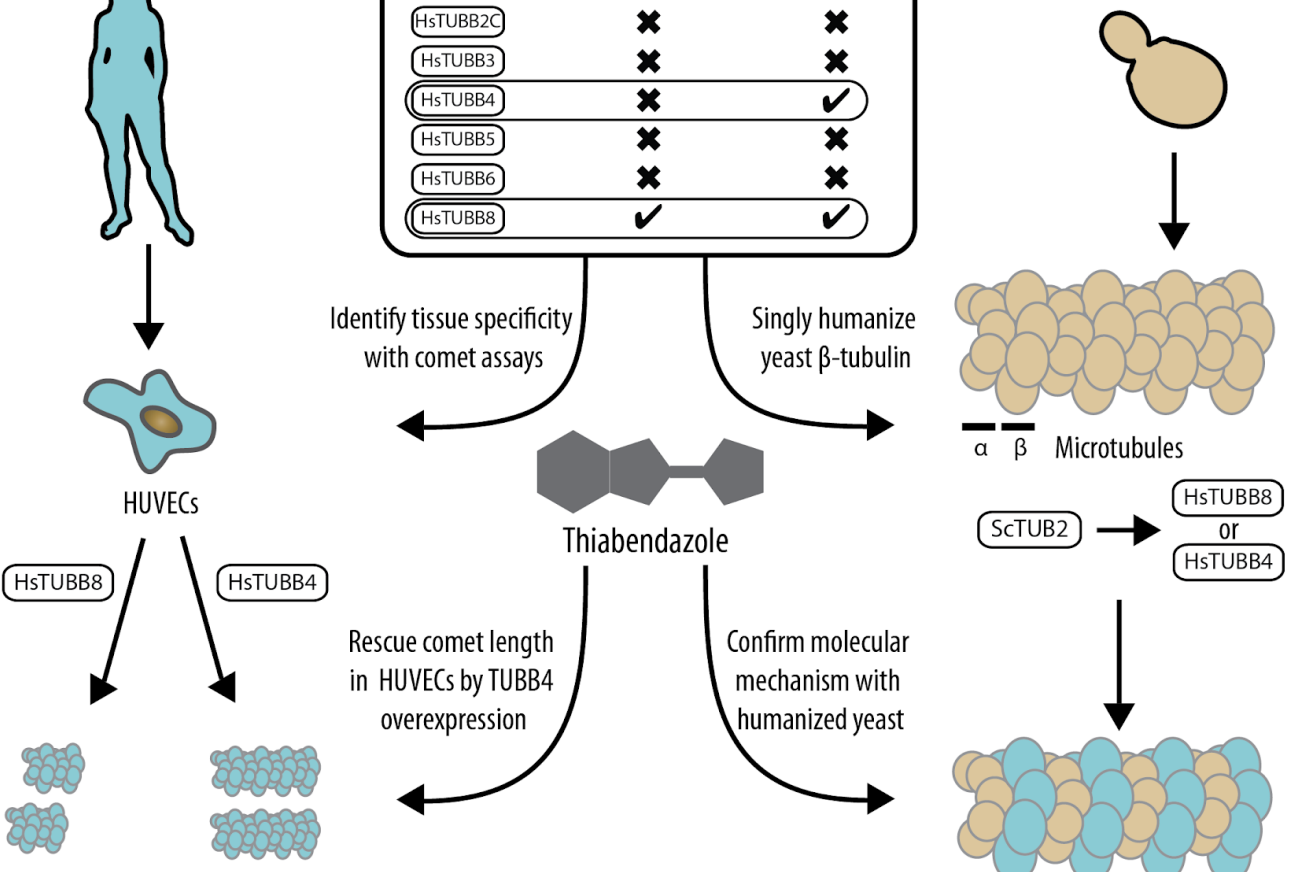
in HUVECs by TUBB4
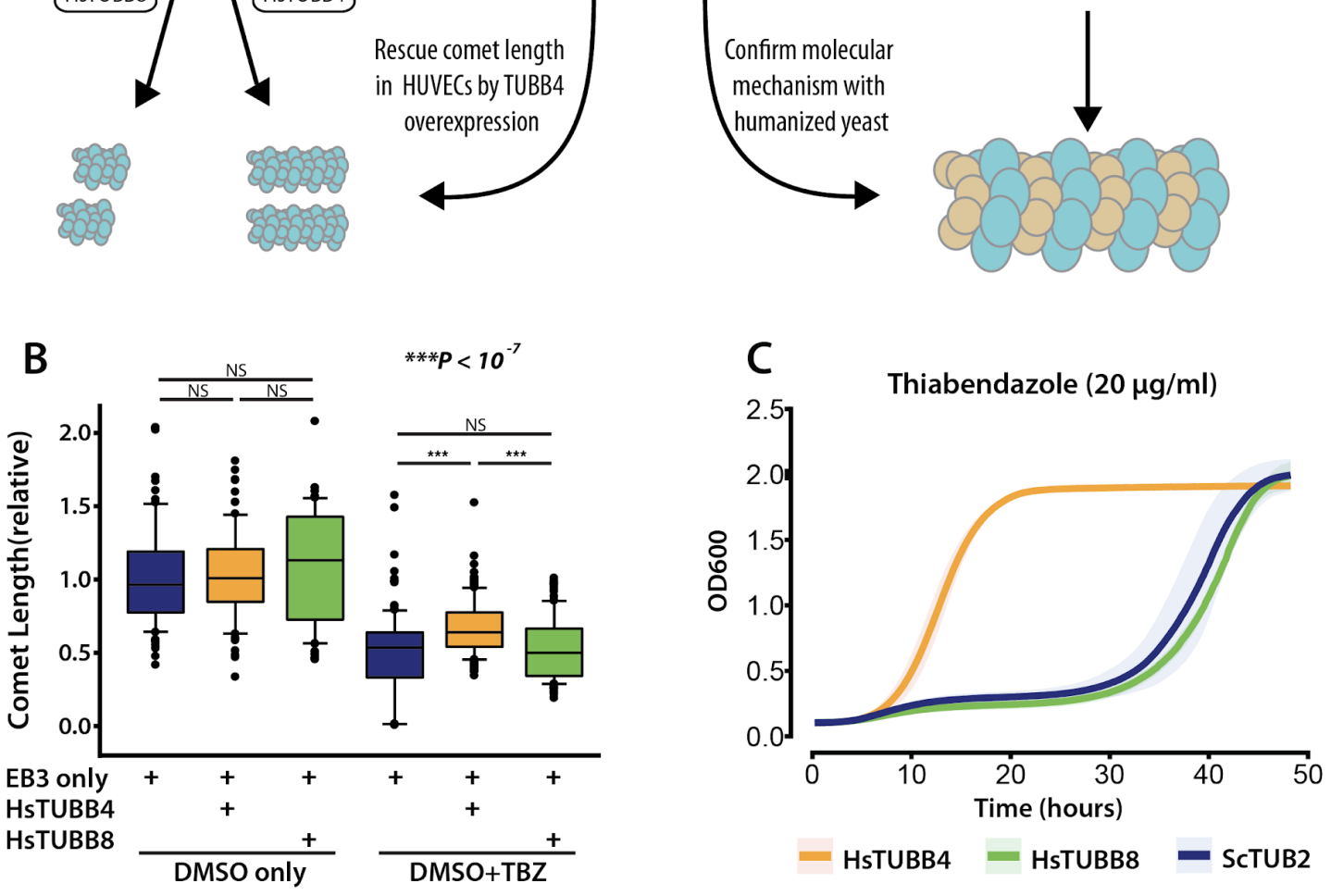

Figure 3. TBZ specifically inhibits the human $\beta$-tubulin TUBB8, not TUBB4, in HUVEC cell culture and humanized yeast . 
A

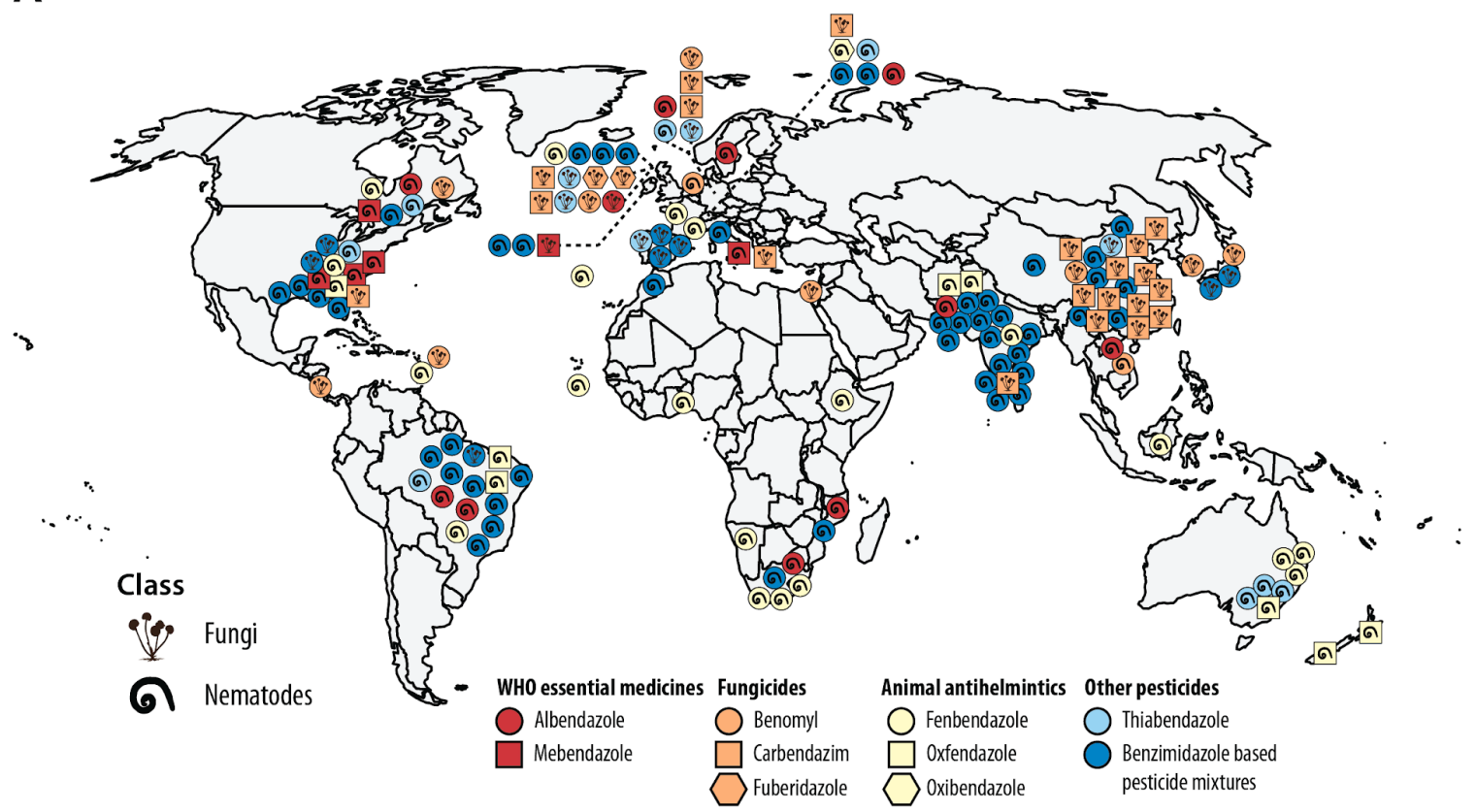

B

Figure 4. Global trends in benzimidazole resistance mutations and chemical structural similarities suggest numerous potential vascular disrupting agents. 

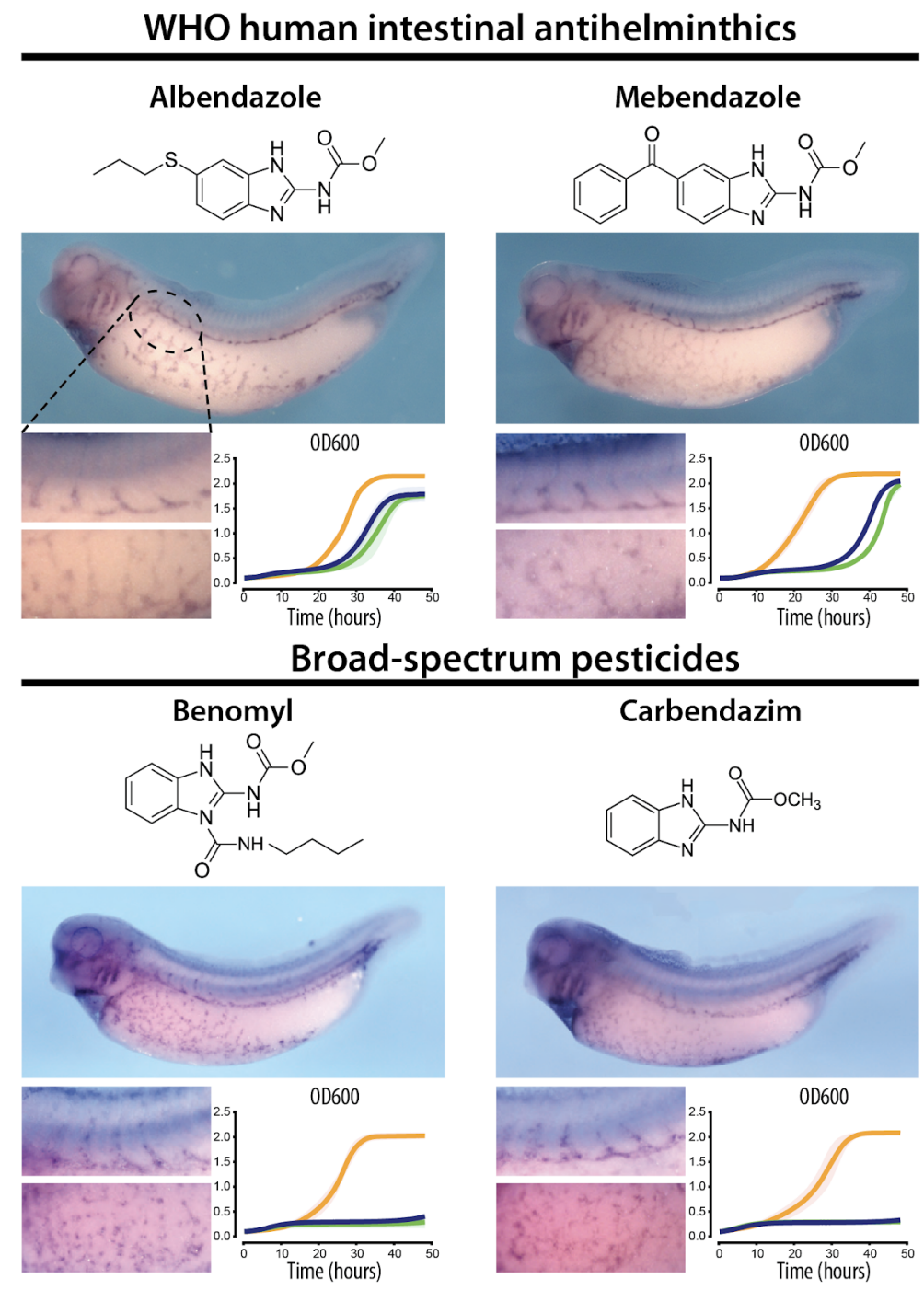

\section{Animal antihelminthic}

Fenbendazole

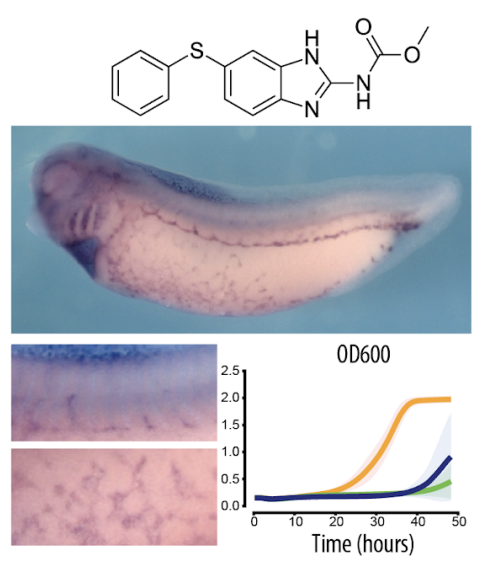

\section{DMSO (control)}

Yeast Strain Legend

- HsTUBB4

- HsTUBB8

- ScTUB2

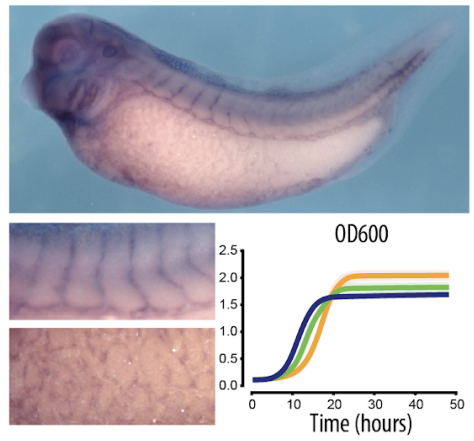

Figure 5. Commercially used benzimidazole pesticides, antifungals, and antihelminthics are also TUBB8-specific and disrupt vasculature. 
bioRxiv preprint doi: https://doi.org/10.1101/2020.09.15.298828; this version posted September 15, 2020. The copyright holder for this preprint (which was not certified by peer review) is the author/funder, who has granted bioRxiv a license to display the preprint in perpetuity. It is made available under aCC-BY 4.0 International license.

A

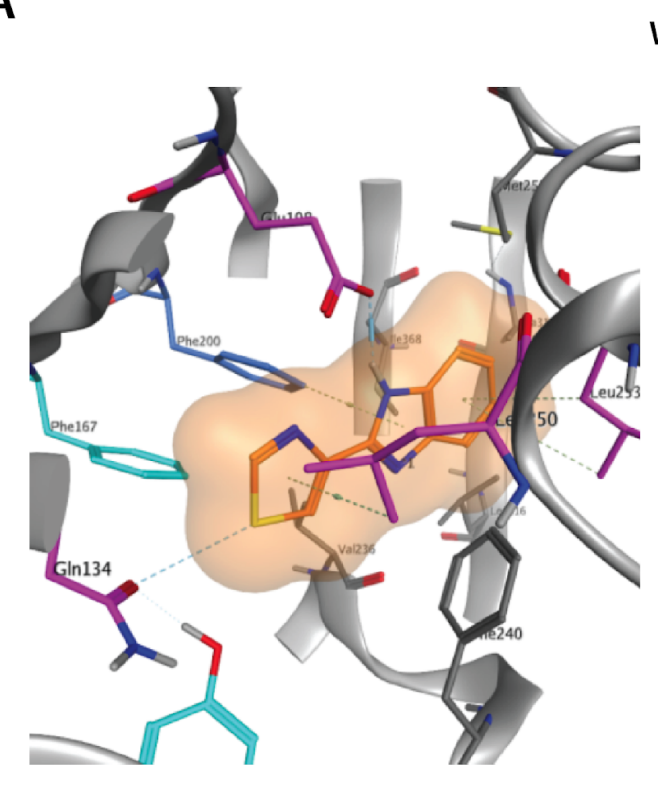

Wild-type

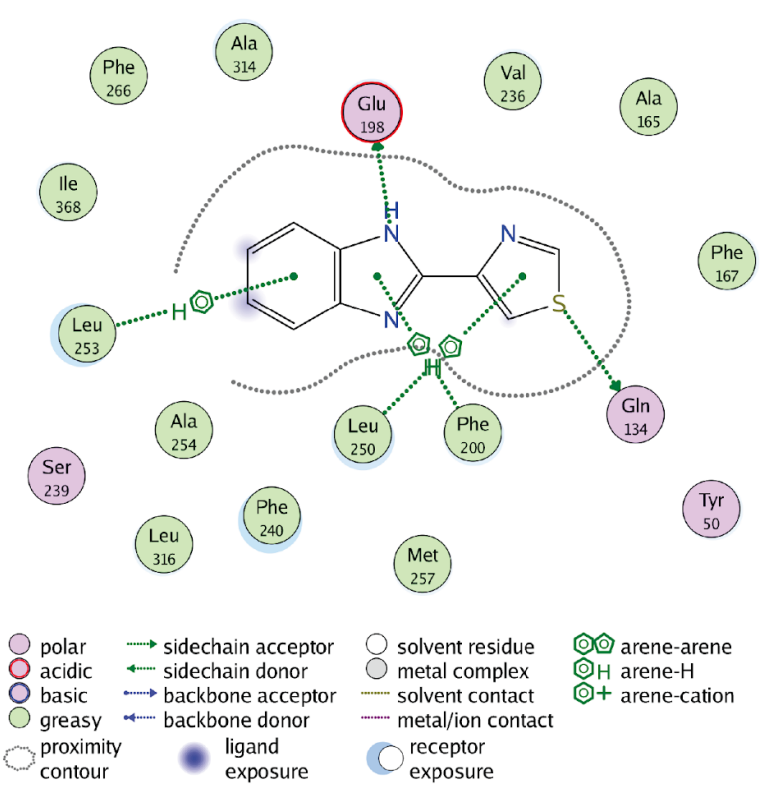

B

F200Y mutant
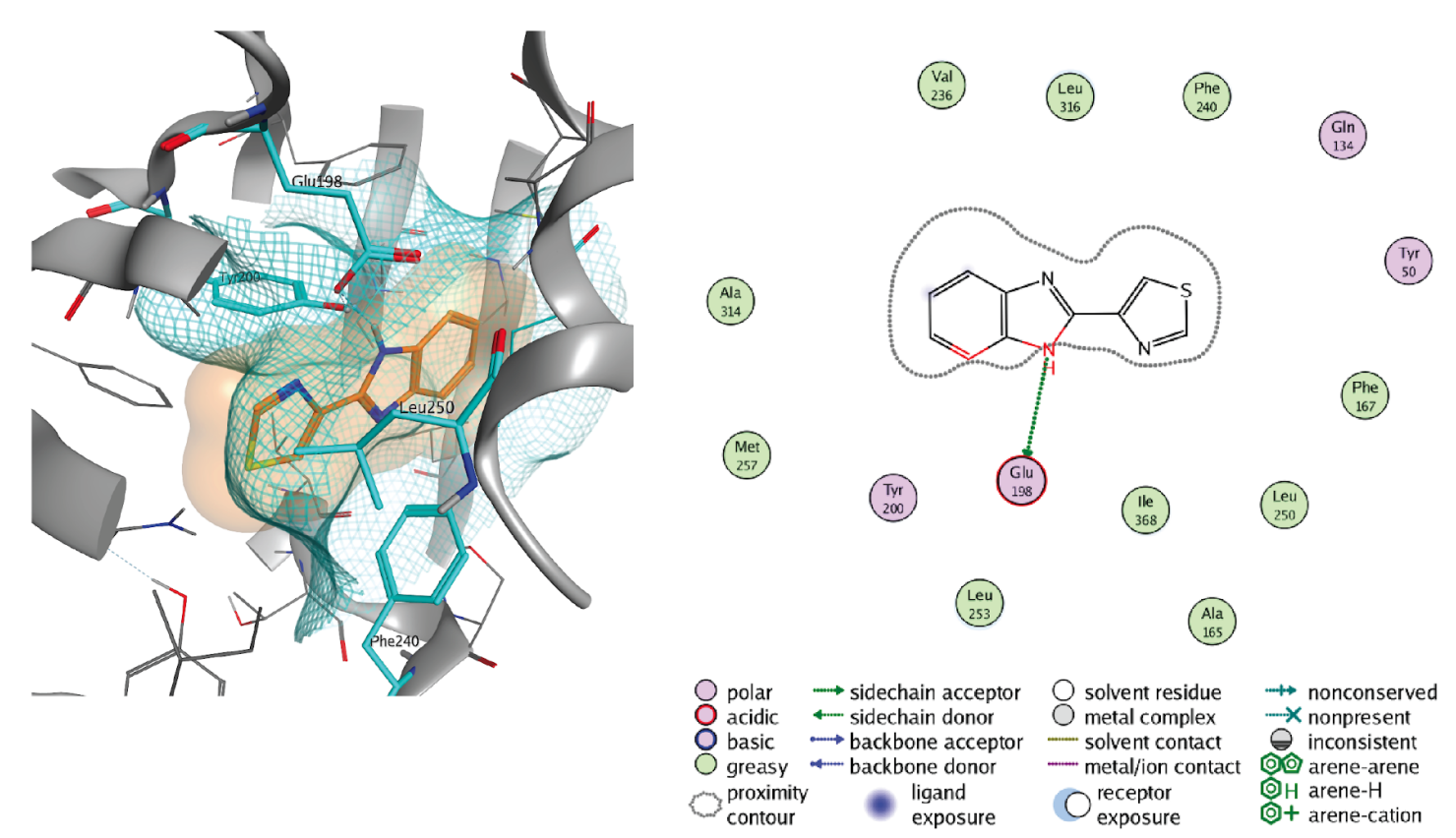

Figure S1. Homology modeling and in silico docking studies predict the TBZ binding site in the fungal $\beta$-tubulin $N D A 3$ structure. 
A

HsTUBB4

$\left(\begin{array}{lll}200 \\ 200\end{array}\right)$

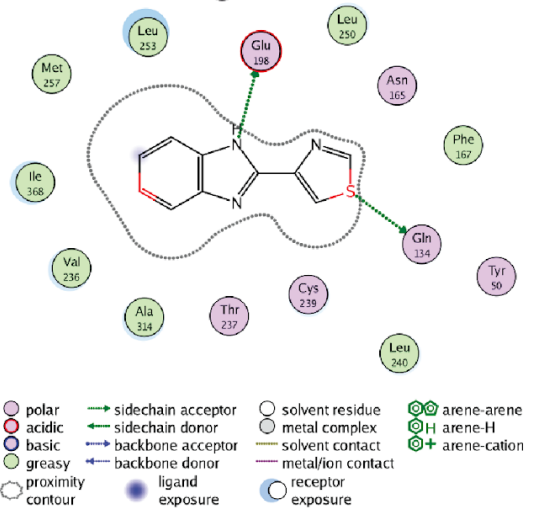

HsTUBB8

$\left(\begin{array}{c}A m 5 \\ 165\end{array}\right)$

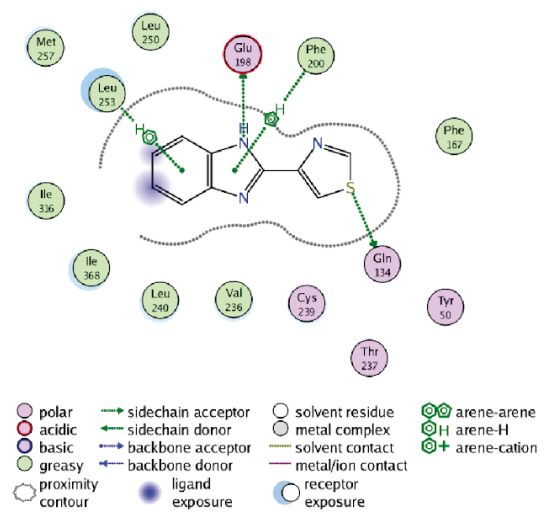

B

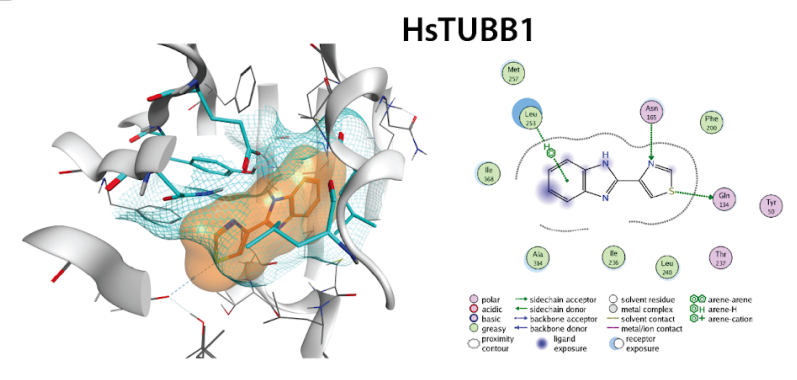

HsTUBB2C
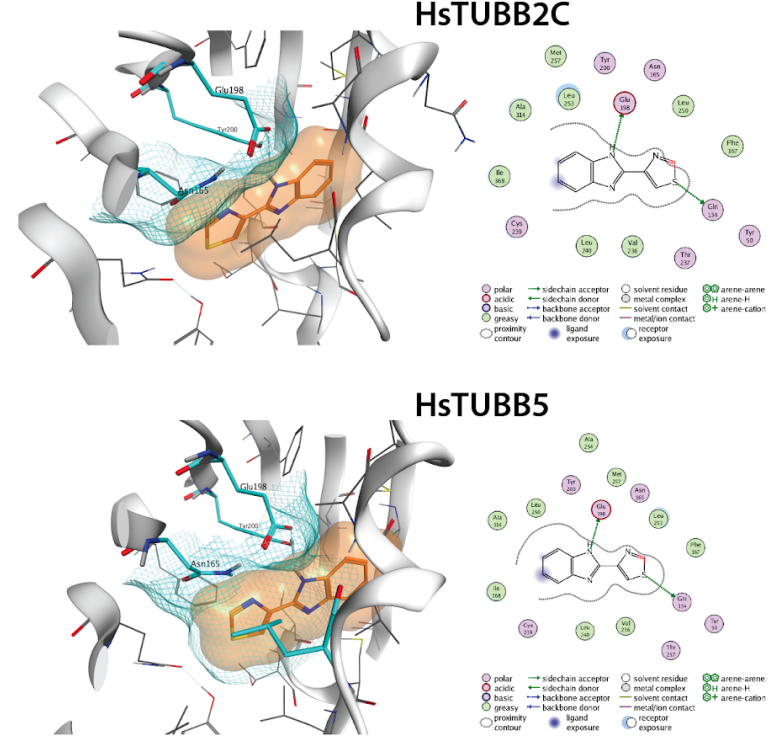

HsTUBB5

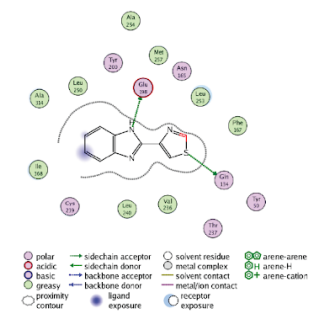

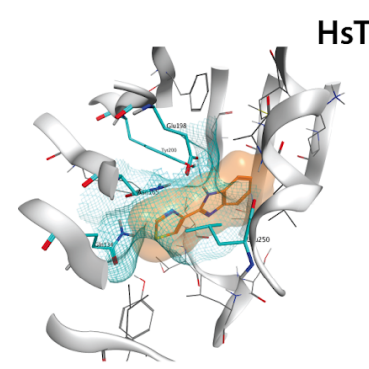

HsTUBB2A
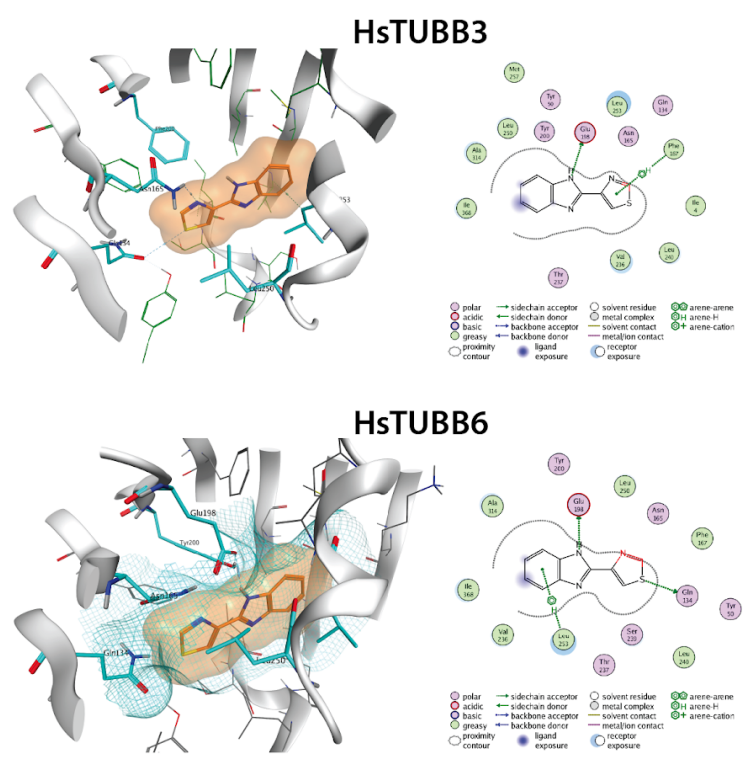

Figure S2. Only TUBB8 favorably binds TBZ among the 9 human $\beta$-tubulins. 
A

Thiabendazole concentration $(\mu \mathrm{g} / \mathrm{ml})$
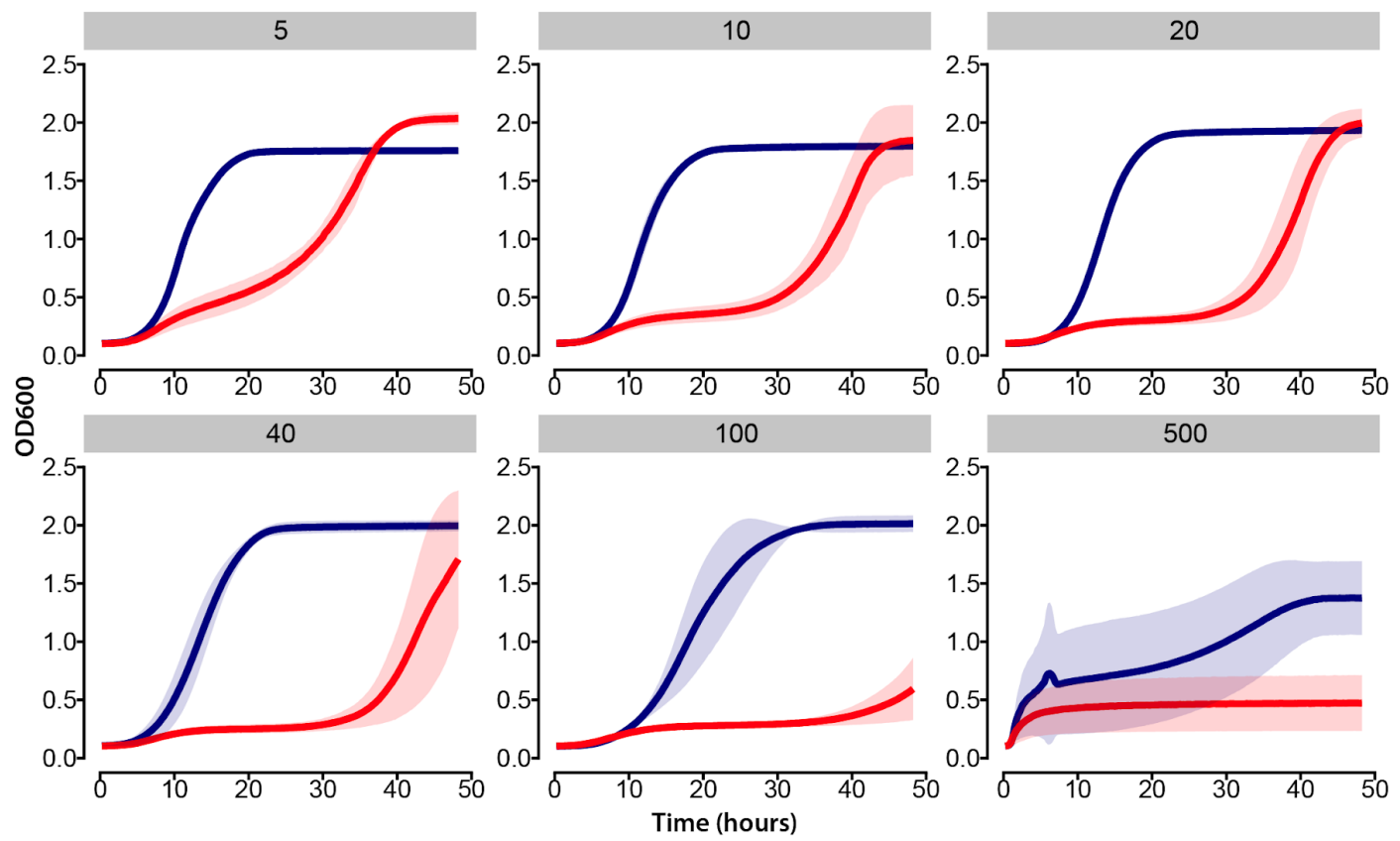

Strain

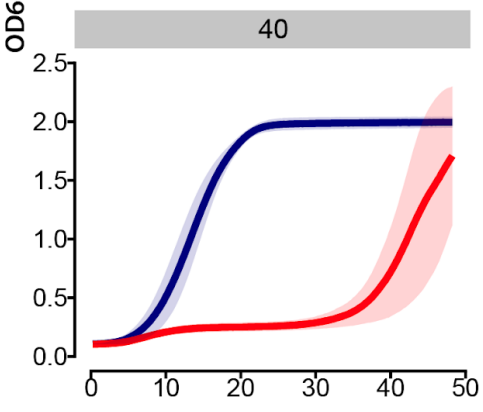

- BY4741

- BY4741tub3A

B
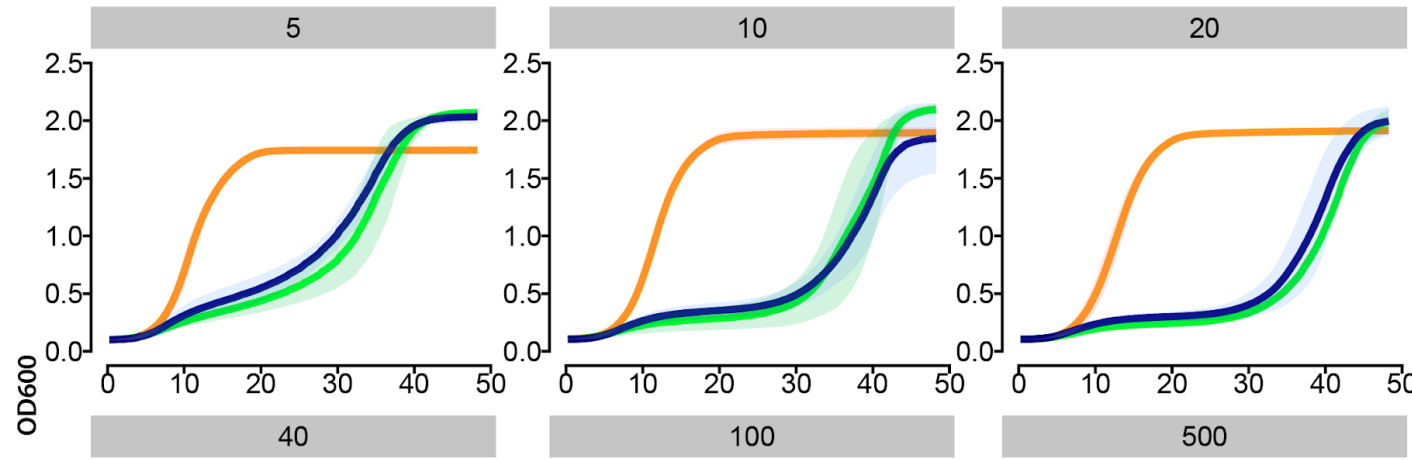

Strain
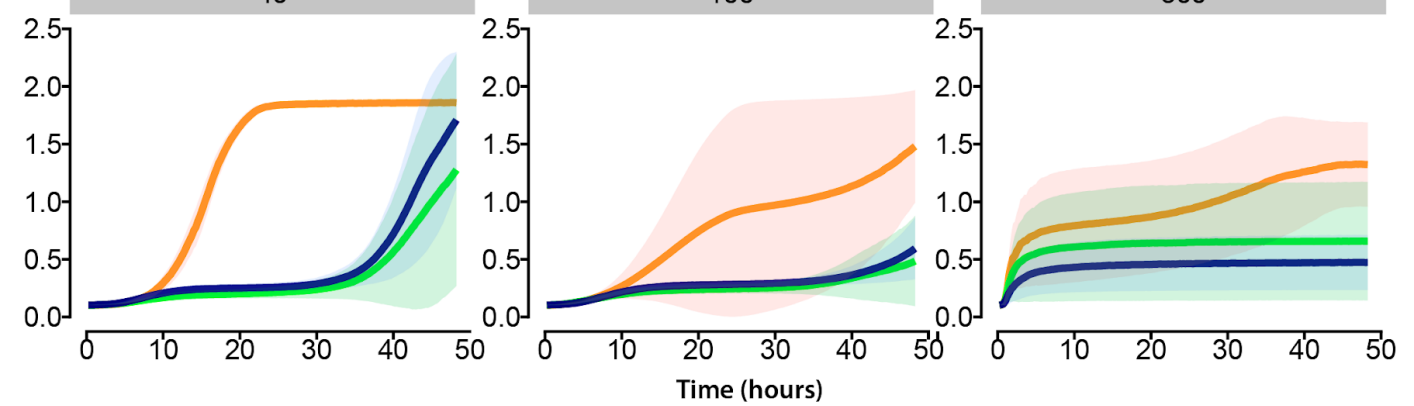

- HsTUBB4

- HsTUBB8

- ScTUB2

Figure S3. Yeast strains with modified $\beta$-tubulin are differentially sensitive to TBZ. 


\section{Albendazole concentration $(\mu \mathrm{g} / \mathrm{ml})$}
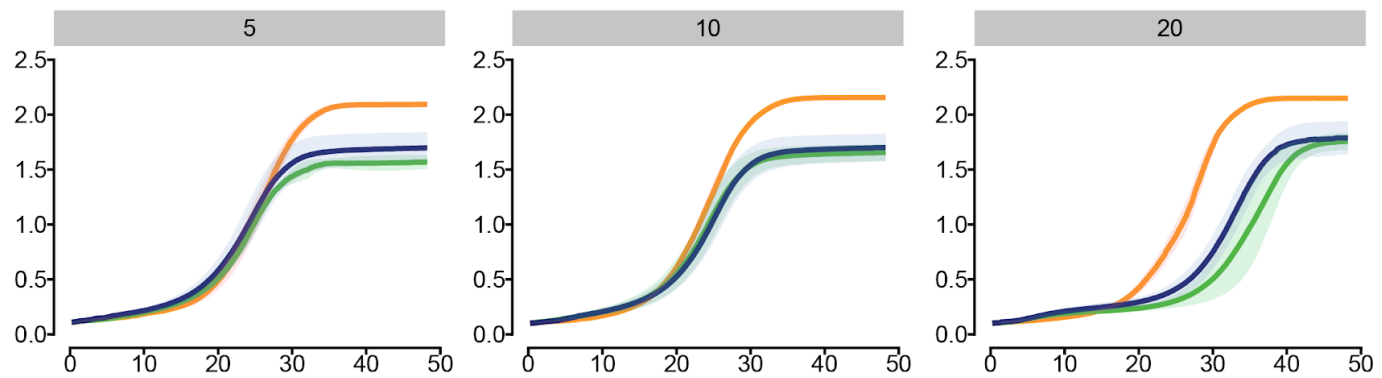

\section{Benomyl concentration $(\mu \mathrm{g} / \mathrm{ml})$}
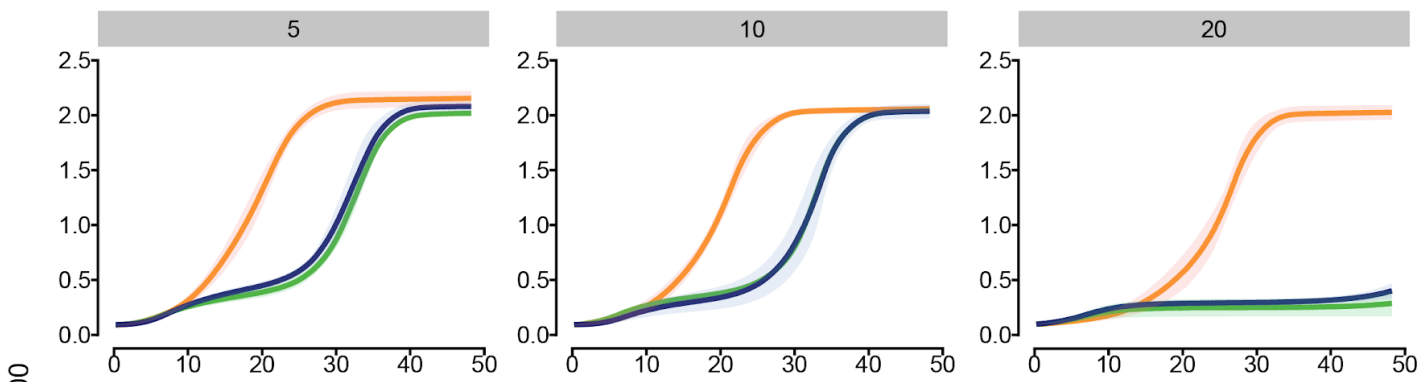

8
$\varnothing$
0

Carbendazim concentration $(\mu \mathrm{g} / \mathrm{ml})$
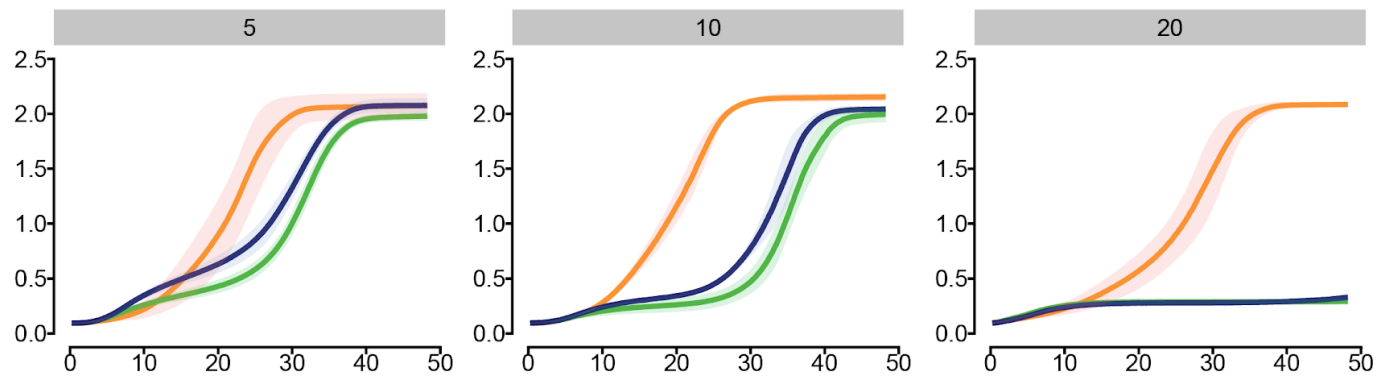

Colchicine concentration $(\mu \mathrm{g} / \mathrm{ml})$
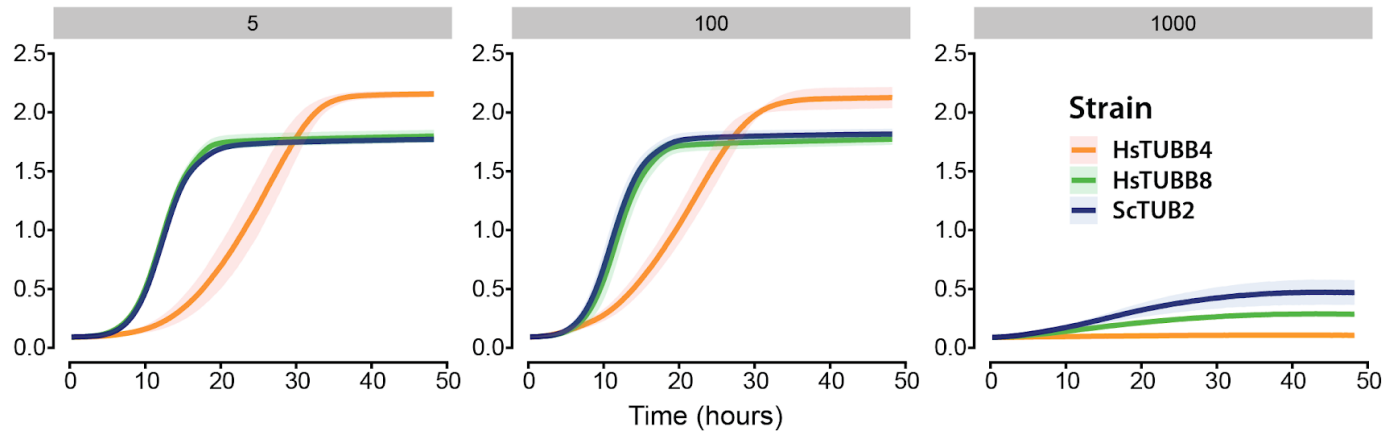

Figure S4. Growth profiles of benzimidazole treated yeast strains. 
A
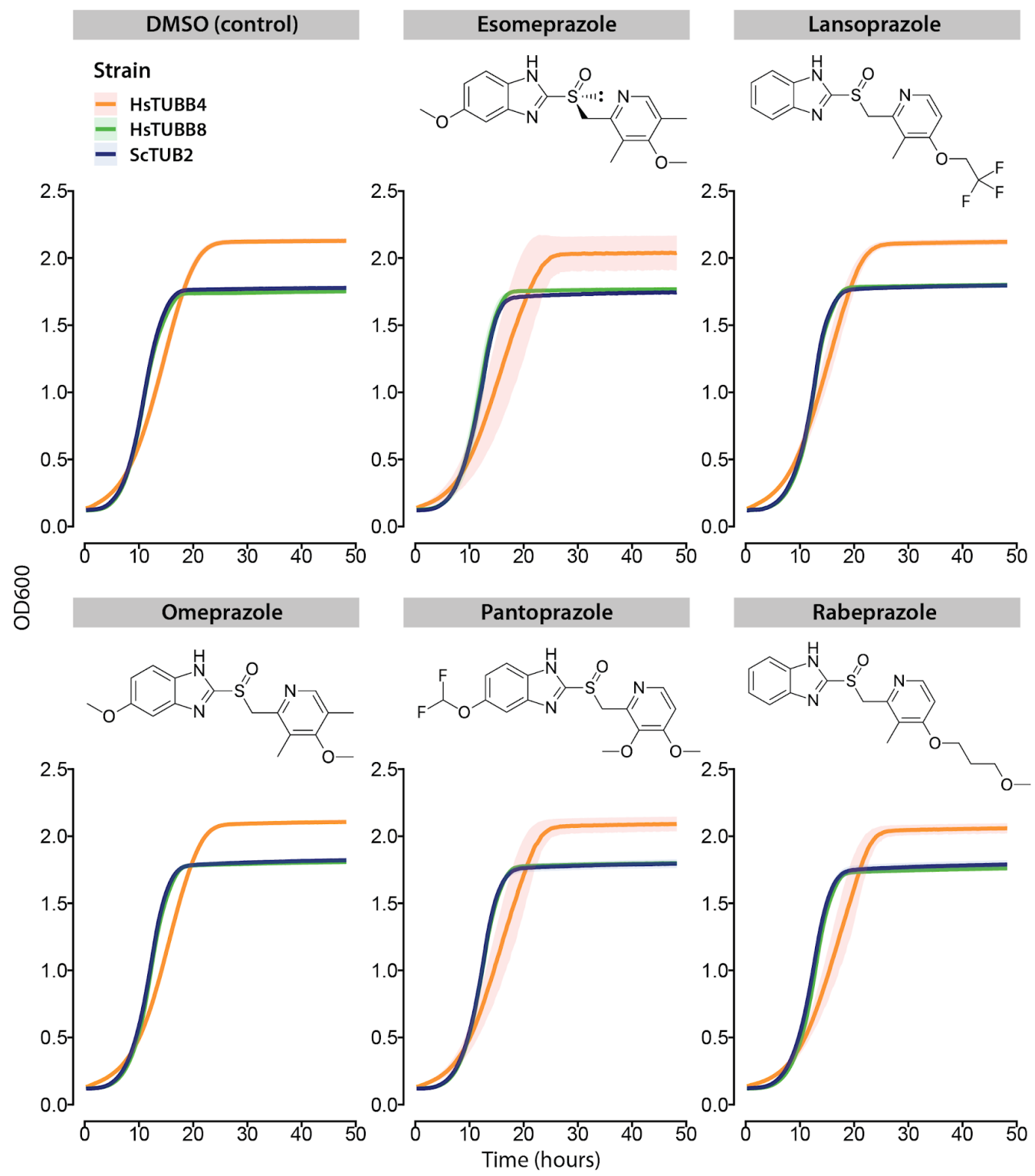

B

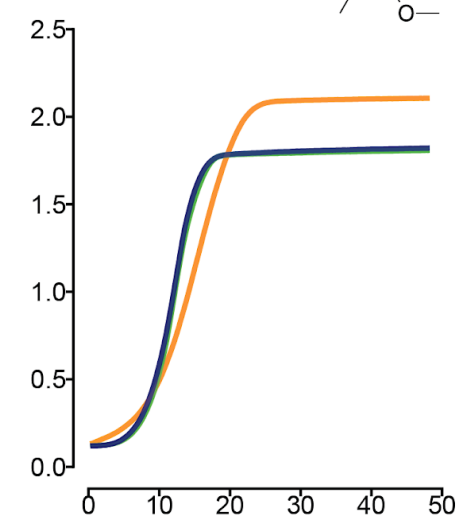

Triclabendazole
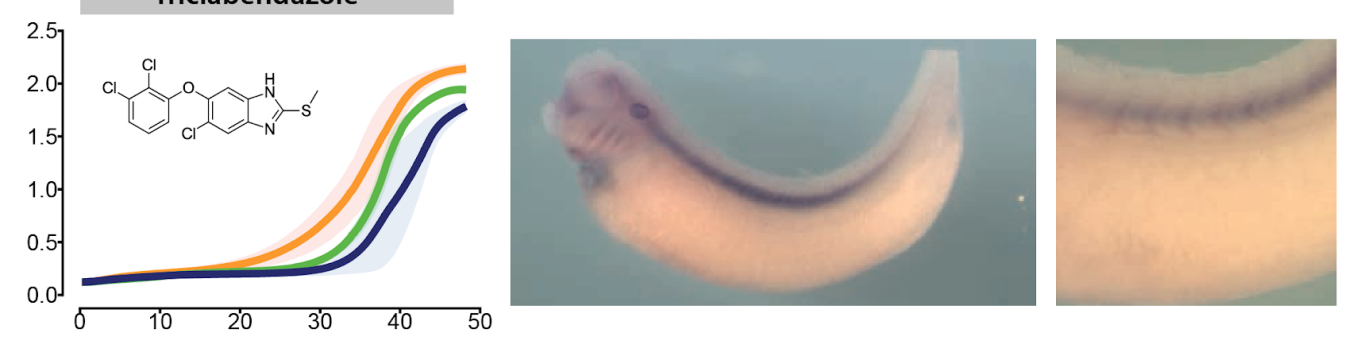

Figure S5. Proton-pump inhibitors do not elicit growth defects in humanized strains. 


\section{References}

539 1. Carmeliet, P. Angiogenesis in life, disease and medicine. Nature 438, 932-936 (2005).

540 2. Herbert, S. P. \& Stainier, D. Y. R. Molecular control of endothelial cell behaviour during

541 blood vessel morphogenesis. Nature Reviews Molecular Cell Biology 12, 551-564 (2011).

542 3. Folkman, J. Opinion: Angiogenesis: an organizing principle for drug discovery? Nature

543 Reviews Drug Discovery 6, 273-286 (2007).

544 4. Kerbel, R. S. Tumor Angiogenesis. New England Journal of Medicine 358, 2039-2049

$545 \quad$ (2008).

546 5. Folkman, J. Endogenous angiogenesis inhibitors. APMIS 112, 496-507 (2004).

547 6. O'Reilly, M. S. et al. Endostatin: an endogenous inhibitor of angiogenesis and tumor growth. $548 \quad$ Cell 88, 277-285 (1997).

549 7. O'Reilly, M. S. et al. Angiostatin: a novel angiogenesis inhibitor that mediates the 550 suppression of metastases by a Lewis lung carcinoma. Cell 79, 315-328 (1994).

551 8. Nyberg, P., Xie, L. \& Kalluri, R. Endogenous Inhibitors of Angiogenesis. Cancer Res 65 , $552 \quad 3967-3979(2005)$.

553 9. El-Kenawi, A. E. \& El-Remessy, A. B. Angiogenesis inhibitors in cancer therapy:

554 mechanistic perspective on classification and treatment rationales. Br J Pharmacol 170, 712$555 \quad 729(2013)$.

556 10. Heath, V. L. \& Bicknell, R. Anticancer strategies involving the vasculature. Nature Reviews 557 Clinical Oncology 6, 395-404 (2009).

558 11. Hinnen, P. \& Eskens, F. a. L. M. Vascular disrupting agents in clinical development. British 559 Journal of Cancer 96, 1159-1165 (2007).

560 12. Mason, R. P., Zhao, D., Liu, L., Trawick, M. L. \& Pinney, K. G. A Perspective on Vascular 561 Disrupting Agents that Interact with Tubulin: Preclinical Tumor Imaging and Biological Assessment. Integr Biol (Camb) 3, 375-387 (2011).

563 13. Lippert, J. W. Vascular disrupting agents. Bioorganic \& Medicinal Chemistry 15, 605-615 564 (2007).

565 14. Nowak-Sliwinska, P., van den Bergh, H., Sickenberg, M. \& Koh, A. H. C. Photodynamic 566 therapy for polypoidal choroidal vasculopathy. Progress in Retinal and Eye Research 37, $567 \quad 182-199(2013)$.

568 15. Ibrahim, M. A. et al. Vascular disrupting agent for neovascular age related macular 
degeneration: a pilot study of the safety and efficacy of intravenous combretastatin a-4 phosphate. BMC Pharmacology and Toxicology 14, 7 (2013).

16. Tozer, G. M., Kanthou, C. \& Baguley, B. C. Disrupting tumour blood vessels. Nat Rev Cancer 5, 423-435 (2005).

17. Cai, S. X. Small molecule vascular disrupting agents: potential new drugs for cancer treatment. Recent Pat Anticancer Drug Discov 2, 79-101 (2007).

18. McGary, K. L. et al. Systematic discovery of nonobvious human disease models through orthologous phenotypes. PNAS 107, 6544-6549 (2010).

19. Woods, J. O., Singh-Blom, U. M., Laurent, J. M., McGary, K. L. \& Marcotte, E. M. Prediction of gene-phenotype associations in humans, mice, and plants using phenologs. BMC Bioinformatics 14, 203 (2013).

20. Cha, H. J. et al. Evolutionarily Repurposed Networks Reveal the Well-Known Antifungal (2012).

21. Taylor, R. D., MacCoss, M. \& Lawson, A. D. G. Rings in Drugs: Miniperspective. J. Med. Chem. 57, 5845-5859 (2014).

22. Skuce, P., Stenhouse, L., Jackson, F., Hypša, V. \& Gilleard, J. Benzimidazole resistance allele haplotype diversity in United Kingdom isolates of Teladorsagia circumcincta supports a hypothesis of multiple origins of resistance by recurrent mutation. International Journal for Parasitology 40, 1247-1255 (2010).

23. Davidse, L. C. \& Flach, W. Interaction of thiabendazole with fungal tubulin. Biochimica et Biophysica Acta (BBA) - General Subjects 543, 82-90 (1978).

24. EPA. Thiabendazole and salts R.E.D Factsheet. United States Environmental Protection Agency https://www.epa.gov/.

25. Lubega, G. W. \& Prichard, R. K. Specific interaction of benzimidazole anthelmintics with tubulin: high-affinity binding and benzimidazole resistance in Haemonchus contortus. Molecular and Biochemical Parasitology 38, 221-232 (1990).

26. Lacey, E. \& Gill, J. H. Biochemistry of benzimidazole resistance. Acta Tropica 56, 245-262 (1994).

27. Aguayo-Ortiz, R. et al. Towards the identification of the binding site of benzimidazoles to $\beta$ tubulin of Trichinella spiralis: Insights from computational and experimental data. Journal of 
Molecular Graphics and Modelling 41, 12-19 (2013).

601

602

603

604

605

606

607

608

609

610

611

612

613

614

615

616

617

618

619

620

621

622

623

624

625

626

627

628

629

630

28. Vela-Corcía, D., Romero, D., Vicente, A. de \& Pérez-García, A. Analysis of $\beta$-tubulincarbendazim interaction reveals that binding site for $\mathrm{MBC}$ fungicides does not include residues involved in fungicide resistance. Scientific Reports 8, 7161 (2018).

29. Driscoll, M., Dean, E., Reilly, E., Bergholz, E. \& Chalfie, M. Genetic and molecular analysis of a Caenorhabditis elegans beta-tubulin that conveys benzimidazole sensitivity. J. Cell Biol. 109, 2993-3003 (1989).

30. Hahnel, S. R. et al. Extreme allelic heterogeneity at a Caenorhabditis elegans beta-tubulin locus explains natural resistance to benzimidazoles. PLOS Pathogens 14, e1007226 (2018).

31. Furtado, L. F. V. \& Rabelo, É. M. L. Molecular analysis of the F167Y SNP in the $\beta$-tubulin gene by screening genotypes of two Ancylostoma caninum populations. Veterinary Parasitology 210, 114-117 (2015).

32. Furtado, L. F. V., Bello, A. C. P. de P., dos Santos, H. A., Carvalho, M. R. S. \& Rabelo, É. M. L. First identification of the F200Y SNP in the $\beta$-tubulin gene linked to benzimidazole resistance in Ancylostoma caninum. Veterinary Parasitology 206, 313-316 (2014).

33. Ramünke, S. et al. Benzimidazole resistance survey for Haemonchus, Teladorsagia and Trichostrongylus in three European countries using pyrosequencing including the development of new assays for Trichostrongylus. International Journal for Parasitology: Drugs and Drug Resistance 6, 230-240 (2016).

34. Yilmaz, E., Ramünke, S., Demeler, J. \& Krücken, J. Comparison of constitutive and thiabendazole-induced expression of five cytochrome P450 genes in fourth-stage larvae of Haemonchus contortus isolates with different drug susceptibility identifies one gene with high constitutive expression in a multi-resistant isolate. International Journal for Parasitology: Drugs and Drug Resistance 7, 362-369 (2017).

35. Zhang, Y.-J. et al. Effect of Carbendazim Resistance on Trichothecene Production and Aggressiveness of Fusarium graminearum. MPMI 22, 1143-1150 (2009).

36. Ali, Q. et al. Emergence and the spread of the F200Y benzimidazole resistance mutation in Haemonchus contortus and Haemonchus placei from buffalo and cattle. bioRxiv 425660 (2018) doi:10.1101/425660.

37. Baltrušis, P., Halvarsson, P. \& Höglund, J. Exploring benzimidazole resistance in Haemonchus contortus by next generation sequencing and droplet digital PCR. International 
Journal for Parasitology: Drugs and Drug Resistance 8, 411-419 (2018).

38. Brown, M. C., Taylor, G. S. \& Epton, H. a. S. Carbendazim resistance in the eyespot pathogen Pseudocercosporella herpotrichoides. Plant Pathology 33, 101-111 (1984).

39. Carter, H. E., Cools, H. J., West, J. S., Shaw, M. W. \& Fraaije, B. A. Detection and molecular characterisation of Pyrenopeziza brassicae isolates resistant to methyl benzimidazole carbamates. Pest Management Science 69, 1040-1048 (2013).

40. Nakaune, R. \& Nakano, M. Benomyl resistance of Colletotrichum acutatum is caused by enhanced expression of $\beta$-tubulin 1 gene regulated by putative leucine zipper protein CaBEN1. Fungal Genetics and Biology 44, 1324-1335 (2007).

41. Tolliver, S. C., Lyons, E. T., Drudge, J. H., Stamper, S. \& Granstrom, D. E. Critical tests of thiabendazole, oxibendazole, and oxfendazole for drug resistance of population-B equine small strongyles (1989 and 1990). Am. J. Vet. Res. 54, 908-913 (1993).

42. Yang, Y. et al. A new point mutation in $\beta 2$-tubulin confers resistance to carbendazim in Fusarium asiaticum. Pesticide Biochemistry and Physiology 145, 15-21 (2018).

43. Zhu, Z.-Q., Zhou, F., Li, J.-L., Zhu, F.-X. \& Ma, H.-J. Carbendazim resistance in field isolates of Sclerotinia sclerotiorum in China and its management. Crop Protection 81, 115121 (2016).

44. Dawson, P. J., Gutteridge, W. E. \& Gull, K. A comparison of the interaction of anthelmintic benzimidazoles with tubulin isolated from mammalian tissue and the parasitic nematode Ascaridia galli. Biochemical Pharmacology 33, 1069-1074 (1984).

45. Davidse, L. C. Benzimidazole Fungicides: Mechanism of Action and Biological Impact. Annual Review of Phytopathology 24, 43-65 (1986).

46. Ranaivoson, F. M., Gigant, B., Berritt, S., Joullié, M. \& Knossow, M. Structural plasticity of tubulin assembly probed by vinca-domain ligands. Acta Crystallogr D Biol Crystallogr $\mathbf{6 8}$, 927-934 (2012).

47. Barbier, P. et al. Stathmin and Interfacial Microtubule Inhibitors Recognize a Naturally Curved Conformation of Tubulin Dimers. J. Biol. Chem. 285, 31672-31681 (2010). with tubulin provide a rationale for drug discovery. The FEBS Journal 283, 102-111 (2016).

49. Leandro-García, L. J. et al. Tumoral and tissue-specific expression of the major human betatubulin isotypes. Cytoskeleton (Hoboken) 67, 214-223 (2010). 
50. Kavallaris, M. Microtubules and resistance to tubulin-binding agents. Nat Rev Cancer 10, 194-204 (2010).

51. Sirajuddin, M., Rice, L. M. \& Vale, R. D. Regulation of microtubule motors by tubulin isotypes and post-translational modifications. Nat. Cell Biol. 16, 335-344 (2014).

52. Garge, R. K., Laurent, J. M., Kachroo, A. H. \& Marcotte, E. M. Systematic Humanization of the Yeast Cytoskeleton Discerns Functionally Replaceable from Divergent Human Genes. Genetics 215, 1153-1169 (2020).

53. Schatz, P. J., Solomon, F. \& Botstein, D. Genetically essential and nonessential alpha-tubulin genes specify functionally interchangeable proteins. Mol. Cell. Biol. 6, 3722-3733 (1986).

54. Liu, S. et al. Carbendazim resistance and dimethachlone sensitivity of field isolates of Sclerotinia sclerotiorum from oilseed rape in Henan Province, China. Journal of Phytopathology 166, 701-708 (2018).

55. Santos, J. M. L. dos et al. Haemonchus contortus $\beta$-tubulin isotype 1 gene F200Y and F167Y

56. Zhang, H. et al. A single-nucleotide-polymorphism-based genotyping assay for simultaneous detection of different carbendazim-resistant genotypes in the Fusarium graminearum species complex. PeerJ 4, e2609 (2016).

57. Kumar, S. et al. Benzimidazole resistance in equine cyathostomins in India. Veterinary Parasitology 218, 93-97 (2016).

58. Rupp, S. et al. Botrytis fragariae, a New Species Causing Gray Mold on Strawberries, Shows High Frequencies of Specific and Efflux-Based Fungicide Resistance. Appl. Environ. Microbiol. 83, (2017).

59. Liu, S., Che, Z. \& Chen, G. Multiple-fungicide resistance to carbendazim, diethofencarb, procymidone, and pyrimethanil in field isolates of Botrytis cinerea from tomato in Henan Province, China. Crop Protection 84, 56-61 (2016).

60. Romero, R. A. \& Sutton, T. B. Characterization of Benomyl Resistance in Mycosphaerella fijiensis, Cause of Black Sigatoka of Banana, in Costa Rica. Plant Disease 82, 931-934 (1998). 
$16627-16638(2015)$

62. Niciura, S. C. M. et al. F200Y polymorphism in the $\beta$-tubulin gene in field isolates of Haemonchus contortus and risk factors of sheep flock management practices related to anthelmintic resistance. Veterinary Parasitology 190, 608-612 (2012).

63. Chagas, A. M. et al. F200Y polymorphism of the $\beta$-tubulin isotype 1 gene in Haemonchus contortus and sheep flock management practices related to anthelmintic resistance in eastern Amazon. Veterinary Parasitology 226, 104-108 (2016).

64. Gossen, B. D., Rimmer, S. R. \& Holley, J. D. First Report of Resistance to Benomyl Fungicide in Sclerotinia sclerotiorum. Plant Disease 85, 1206-1206 (2001).

65. Keegan, J. D., Good, B., de Waal, T., Fanning, J. \& Keane, O. M. Genetic basis of benzimidazole resistance in Teladorsagia circumcincta in Ireland. Irish Veterinary Journal 70, 8 (2017).

66. Banno, S. et al. Genotyping of Benzimidazole-Resistant and Dicarboximide-Resistant Mutations in Botrytis cinerea Using Real-Time Polymerase Chain Reaction Assays. Phytopathology 98, 397-404 (2008).

67. Middelberg, A. \& McKenna, P. B. Oxfendazole resistance in Nematodirus spathiger. New Zealand Veterinary Journal 31, 65-66 (1983).

68. Saeed, M., Iqbal, Z. \& Jabbar, A. Oxfendazole Resistance in Gastrointestinal Nematodes of Beetal Goats at Livestock Farms of Punjab (Pakistan). Acta Vet. Brno 76, 79-85 (2007).

69. Cabañas, R., Castellá, G., Abarca, M. L., Bragulat, M. R. \& Cabañes, F. J. Thiabendazole resistance and mutations in the $\beta$-tubulin gene of Penicillium expansum strains isolated from apples and pears with blue mold decay. FEMS Microbiol Lett 297, 189-195 (2009).

721 framework for R. Bioinformatics 24, 1733-1734 (2008).

722 73. Backman, T. W. H., Cao, Y. \& Girke, T. ChemMine tools: an online service for analyzing and clustering small molecules. Nucleic Acids Res 39, W486-W491 (2011). 
724 74. Uhlén, M. et al. Tissue-based map of the human proteome. Science 347, 1260419 (2015).

725 75. UniProt: a worldwide hub of protein knowledge. Nucleic Acids Res 47, D506-D515 (2019).

726 76. Burga, A., Casanueva, M. O. \& Lehner, B. Predicting mutation outcome from early

727 stochastic variation in genetic interaction partners. Nature 480, 250-253 (2011).

728 77. O’Neil, N. J., Bailey, M. L. \& Hieter, P. Synthetic lethality and cancer. Nature Reviews

729 Genetics 18, 613-623 (2017).

730 78. WHO | Deworming. World Health Organization

731 https://www.who.int/elena/titles/full_recommendations/deworming/en/.

732 79. WORLD HEALTH ORGANIZATION. GUIDELINE: preventive chemotherapy to control

733 soil-transmitted helminth infections in at-risk... population groups. (WORLD HEALTH

734 ORGANIZATION, 2018).

735 80. Laurent, J. M., Young, J. H., Kachroo, A. H. \& Marcotte, E. M. Efforts to make and apply

736 humanized yeast. Brief Funct Genomics 15, 155-163 (2016).

737 81. Laurent, J. M. et al. Humanization of yeast genes with multiple human orthologs reveals

738 functional divergence between paralogs. PLOS Biology 18, e3000627 (2020).

739 82. Kachroo, A. H. et al. Systematic humanization of yeast genes reveals conserved functions

$740 \quad$ and genetic modularity. Science 348, 921-925 (2015).

741 83. Katoh, K. \& Standley, D. M. MAFFT Multiple Sequence Alignment Software Version 7:

742 Improvements in Performance and Usability. Molecular Biology and Evolution 30, 772-780

743 (2013).

744 84. Labute, P. Protonate3D: Assignment of ionization states and hydrogen coordinates to

745 macromolecular structures. Proteins 75, 187-205 (2009).

746 85. Aguayo-Ortiz, R. et al. Molecular basis for benzimidazole resistance from a novel $\beta$-tubulin

747 binding site model | Elsevier Enhanced Reader. Journal of Molecular Graphics and

$748 \quad$ Modelling doi:https://doi.org/10.1016/j.jmgm.2013.07.008.

749 86. Labute, P. LowModeMD_-Implicit Low-Mode Velocity Filtering Applied to

750 Conformational Search of Macrocycles and Protein Loops. J. Chem. Inf. Model. 50, 792-800

751 (2010).

752 87. Clark, A. M. \& Labute, P. 2D Depiction of Protein-Ligand Complexes. J. Chem. Inf. Model.

$753 \quad 47,1933-1944(2007)$.

754 88. Stepanova, T. et al. Visualization of Microtubule Growth in Cultured Neurons via the Use of 
EB3-GFP (End-Binding Protein 3-Green Fluorescent Protein). J. Neurosci. 23, 2655-2664 (2003).

757

89. Kim, S. et al. PubChem Substance and Compound databases. Nucleic Acids Res 44, D1202D1213 (2016).

90. O’Boyle, N. M. et al. Open Babel: An open chemical toolbox. Journal of Cheminformatics

$760 \quad 3,33(2011)$.

761 91. Akhmetov, A. et al. Single-step Precision Genome Editing in Yeast Using CRISPR-Cas9.

762 BIO-PROTOCOL 8, (2018).

763 92. Lee, M. E., DeLoache, W. C., Cervantes, B. \& Dueber, J. E. A Highly Characterized Yeast

764 Toolkit for Modular, Multipart Assembly. ACS Synth. Biol. 4, 975-986 (2015).

765 93. Akhmetov, A. et al. Single-step Precision Genome Editing in Yeast Using CRISPR-Cas9.

766 Bio Protoc 8, (2018).

767 94. Lamesch, P. et al. hORFeome v3.1: A resource of human open reading frames representing 768 over 10,000 human genes. Genomics 89, 307-315 (2007). 